\title{
Molecular interaction mechanism of a 14-3-3 protein with a phosphorylated peptide elucidated by enhanced conformational sampling
}

\author{
Junichi Higo,,' Takeshi Kawabata, ${ }^{2}$ Ayumi Kusaka,' Kota Kasahara, ${ }^{3}$ Narutoshi Kamiya,' Ikuo \\ Fukuda, ${ }^{,}$Kentaro Mori, ${ }^{1,4}$ Yutaka Hata,' Yoshifumi Fukunishi, ${ }^{5}$ Haruki Nakamura ${ }^{2}$
}

Graduate School of Simulation Studies, University of Hyogo, 7-1-28 Minatojima Minami- machi, Chuo-ku, Kobe, Hyogo 650-0047, Japan

${ }^{2}$ Institute for Protein Research, Osaka University, 3-2 Yamada-oka, Suita, Osaka 565-0871, Japan

${ }^{3}$ College of Life Sciences, Ritsumeikan University, 1-1-1 Noji-higashi, Kusatsu, Shiga 525-8577, Japan

${ }^{4}$ Maizuru College, 234 Shiroya, Maizuru, Kyoto, 625-8511 Japan

"Cellular and Molecular Biotechnology Research Institute, National Institute of Advanced Industrial Science and Technology (AIST), 2-3-26, Aomi, Koto-ku, Tokyo, 135-0064, Japan

\begin{abstract}
Enhanced conformational sampling, a genetic-algorithm-guided multi-dimensional virtual-system coupled molecular dynamics, can provide equilibrated conformational distributions of a receptor protein and a flexible ligand at room temperature. The distributions provide not only the most stable but also semi-stable complex structures, and propose a ligand-receptor binding process. This method was applied to a system consisting of a receptor protein, 14-3-3e, and a flexible peptide, phosphorylated Myeloid leukemia factor 1 (pMLF1). The results present comprehensive binding pathways of pMLF1 to $14-3-3 \varepsilon$. We identified four thermodynamically stable clusters of MLF1 on the 14-3-3 $\varepsilon$ surface, and free-energy barriers among some clusters. The most stable cluster includes two high-density spots connected by a narrow corridor. When pMLF1 passes the corridor, a salt-bridge relay (switching) related to the phosphorylated residue of pMLF1 occurs. Conformations in one high-density spots are similar to the experimentally determined complex structure. Three-dimensional distributions of residues in the intermolecular interface rationally explain the binding-constant changes resultant from alanine-mutation experiment for the residues. We performed a simulation of non-phosphorylated peptide and 14-3-3e, which demonstrated that the complex structure was unstable, suggesting that phosphorylation of the peptide is crucially important for binding to $14-3-3 \varepsilon$.
\end{abstract}




\section{INTRODUCTION}

The 14-3-3 proteins are eukaryotic adapter proteins that bind short phosphorylated sequences (14-3-3 interaction sequences).-1-3 They bind to numerous partner proteins, usually by recognition of a phosphoserine or phosphothreonine motif. Although several binding motif patterns have been proposed for 14-3-3 interaction sequences, the mechanism of their binding specificities is not understood clearly yet, except for salt bridges between the phosphorylated site of 14-3-3 interaction sequences and 14-3-3 proteins.

Binding of 14-3-3 proteins and 14-3-3 interaction sequences can regulate the subcellular localization and enzymatic activities of proteins related to signal transduction and cell-cycle regulation. ${ }^{4}$ Therefore, 14-3-3 proteins are well known as potential drug targets. Small molecules such as peptide mimetic and natural products have been explored to elucidate inhibitors and stabilizers of 14-3-3 protein complexes. ${ }^{5}$

Humans have seven 14-3-3 proteins that are mutually similar: $\beta, \gamma, \varepsilon, \eta, \sigma, \tau$, and $\zeta$. Among them, the sequence identities are higher than 60\%. After we analyzed the 14-3-3 interaction sequences that bind to the human $14-3-3 \varepsilon$ and $\zeta$ proteins using MATRAS software, ${ }^{6}$ we presented the aligned sequence in Figures S1A and S1B of Supporting Information (SI). The figures portray S1C and S1D, displaying the superimposed structures of the 14-3-3 interaction sequences binding to $14-3-3 \varepsilon$ and $14-3-3 \zeta$ proteins from two orientations. Figure S1E shows the RMSD of the superimposed structures at each residue site. Although some diversity exists in the sequences (Figures S1A and S1B), Figures S1C-E show that the binding structures of the 14-3-3 interaction sequences have similarity around the phosphorylated residue. Therefore, if the complex-formation mechanisms of a 14-3-3 protein and an interaction sequence are understood using a computational method, then this mechanism would be useful for understanding other 14-3-3 complexes.

Molzan et al. solved an X-ray crystal structure (PDB ID: 3ual) (Figure S2) of the human 14-3$3 \varepsilon$ protein bound to a phosphorylated peptide (sequence $=$ RSFpSEPFG, where $\mathrm{pS}$ is a phosphorylated serine at the 34th residue position) taken from myeloid leukemia factor 1 (MLF1).? They also measured the dissociation constants $K_{D}$ of alanine-mutated MLF1 peptides at the 33rd, 35th, and 37th residues (F33A, E35A, and F37A) using isothermal titration calorimetry (ITC). It is particularly interesting that only the mutation F33A led to a significant decrease in affinity, which might be attributable to the loss of hydrophobic interactions by Phe 33 sandwiched between two hydrophobic residues Leu 223 and Leu 230 of the 14-3-3e protein (Figure S2). In contrast, E35A and F37A exhibited almost no change in $K_{D}$. Phe 37 is located on the surface of 14-3-3e in the complex forming a weak hydrophobic contact to the stem of Lys 40 of 14-3-3e. This can be a reason for the negligibly small change of $K_{D}$ by F37A. In the crystal structure, however, Glu 35 is located at a position that can form salt bridges to Lys 50 and Lys 123 of 14-3-3e. Therefore, explaining the reason for the small change of $K_{D}$ by E35A based on the crystal structure is difficult.

The 14-3-3 proteins are related to many human diseases. Kimura et al. reported that a mutation of nuclear distribution E homolog 1 (NDE1) has significant association with schizophrenia. ${ }^{8}$ The 14-3-3 $\varepsilon$ protein bound to the mutated site of NDE1. The 14-3-3 proteins bind to myeloid leukemia factor 1 (MLF1), which is reportedly overexpressed in acute myeloid leukemia.-11 Although Winteringham et al. (2006) reported that the nuclear content of mouse MLF1 was regulated by 143-3 binding," Molzan and Ottmann (2013) reported that the subcellular localization of human MLF1 is independent of 14-3-3 proteins..$^{12}$ Therefore, the biological role of 14-3-3e/MLF1 remains unknown. However, this complex is an appropriate system for the study of complex-formation processes between 14-3-3 proteins and the 14-3-3 interaction sequences comprehensively by 
computations because, as described above, the tertiary structure of 14-3-3e/MLF1 was well studied. The amino-acid contribution to the complex stability was also measured. We infer that, if the computations can explain the measured amino-acid contribution to the complex stability consistently, then the comprehensive binding mechanism is reliable.

Generalized ensemble methods such as the multicanonical sampling, ${ }^{13-16}$ replica exchange ${ }^{17,18}$ method, and their variants ${ }^{19-25}$ are powerful methods to sample large-scale motions of a molecular system by enhancing the conformational motions along the energy or temperature axis. However, these methods might overlook less-stable conformations (i.e., conformations in minor basins) when the minor basins overlap to the major basins in the axis. ${ }^{26.27}$ This oversight is not problematic when the minor basins are beyond the scope of the study. However, when the minor basins act as bridges to connect the major basins, then the oversight might cause conformation trapping, which hinders sampling of a wide conformational space. Consequently, an alternative computational approach must be used.

Adaptive umbrella sampling ${ }^{2829}$ might avoid such an oversight or trapping because one can set a reaction coordinate so that the major and minor basins are discriminated along the reactioncoordinate axis. However, this method requires fine tuning of a weight function (bias function). Practically, the difficulty of tuning might require a very long simulation (or increment of the number of iterative simulations) to treat a complicated system.

Recently, we introduced a method, virtual-system coupled sampling method ${ }^{30}$ and combined it to multicanonical ${ }^{31}$ and adaptive umbrella sampling. ${ }^{2632}$ These methods provide an equilibrated conformational ensemble of the system..$^{33-37}$ Nevertheless, they still require fine tuning of the bias function. We introduced a new method, multi-dimensional virtual-system coupled molecular dynamics (mD-VcMD) ${ }^{3839}$ which is free from fine tuning of the bias function. Furthermore, we extended $\mathrm{mD}-\mathrm{VcMD}$ using a genetic algorithm and designated it as genetic algorithm-guided $\mathrm{mD}$ $\mathrm{VcMD}$ (GA-guided mD-VcMD). ${ }^{40}$

For this study, we performed GA-guided $\mathrm{mD}-\mathrm{VcMD}$ simulations of a system consisting of the receptor (14-3-3e protein) and the ligand (MLF1 segment) to obtain the free-energy landscape which controls ligand-receptor binding. MLF1 was positioned distant from the binding site of 14$3-3 \varepsilon$ in the initial conformations of simulation. MLF1 of two types were examined: phosphorylated and non-phosphorylated MLF1. We examined the contributions of Phe 33, Sep 34, Glu 35, and Phe 37 of MLF1 to the complex stability by analyzing conformational distributions obtained from the simulation, and calculated the free-energy landscape (3D conformational distribution) to discuss comprehensive binding processes of MLF1 to 14-3-3e.

\section{METHODS}

\section{Conformational ensemble from GA-guided mD-VcMD}

To sample both the unbound and bound states of the receptor-ligand system, we use one of generalized ensemble methods: GA-guided $\mathrm{mD}-\mathrm{VcMD}$, which is an extension of $\mathrm{mD}-\mathrm{VcMD}^{38,39}$. Methodological details are explained elsewhere, ${ }^{40}$ and actual parameter values used for the simulation are described in section 4 of SI. Here, only a brief outline of the method is introduced below. This method controls the system's motions in a multi-dimensional $(\mathrm{mD})$ reactioncoordinate (RC) space in general; three RCs, denoted as $\lambda^{(h)}(h=\alpha, \beta, \gamma)$, are introduced for this study. Consequently, "multi-dimensional (mD)" means three-dimensional (3D) for these discussions. The definition of an RC is presented in section 1 of SI. The actual set of the three RCs is given later. 
The outline of the method is as follows. The entire conformational space is first divided into many pieces of local spaces as the particular restricted regions defined by the RCs. In every restricted local conformational space, MD simulation is performed independently at an identical constant temperature. This simulation in the local space is called as the Virtual-state coupled MD (VcMD) simulation. ${ }^{38,39}$ Each local space is overlapped with the neighboring local spaces, and after a particular short period of the MD simulation, the system can transit to the neighboring local spaces depending on the transition probabilities between the overlapped local spaces. The transition probabilities can be set tentatively to appropriate values. Then, after updating the transition probabilities, $\mathrm{VcMD}$ simulations are performed in the next short period, and this procedure is continued until resultant probabilities assigned to the local spaces converge to a stationary distribution. Finally, one can calculate the canonical distribution for the entire RC space from the resultant stationary distribution. Because the iterative procedure used in the original $\mathrm{mD}$ $\mathrm{VcMD}^{38,39}$ is time consuming to attain a convergence for a complicated system with many local minima in the free energy landscape, we developed a new procedure named as the GA-guided mDVcMD, where the Genetic Algorithm is applied to accelerate attaining the stationary distribution even for the complicated system. ${ }^{40}$

The GA-guided mD-VcMD produces a canonical ensemble: a thermally equilibrated conformational ensemble at a simulation temperature. We set the simulation temperature to $300 \mathrm{~K}$ for this study and assigned thermodynamic weight $w_{i}$ to each snapshot, where $i$ represents the $i$ th snapshot in the ensemble. Therefore, we can calculate the distribution function of a quantity at equilibrium if the quantity is expressed by the system conformation.

\section{Systems studied}

We examined two systems in this study: pMLF1/14-3-3 $\varepsilon$ and npMLF1/14-3-3 $\varepsilon$ systems. The former system consists of 14-3-3e and pMLF1 (MLF1 phosphorylated at the 34th serine residue: Ace-Arg[31]-Ser[32]-Phe[33]-Sep[34]-Glu[35]-Pro[36]-Phe[37]-Gly[38]-Nme, where Sep represents a phosphorylated serine, and where Ace and Nme are the acetyl and N-methyl groups, respectively); the latter represents $14-3-3 \varepsilon$ and npMLF1 (MLF1 non-phosphorylated at the 34th serine residue of Ace-Arg[31]-Ser[32]-Phe[33]-Ser[34]-Glu[35]-Pro[36]-Phe[37]-Gly[38]-Nme). The biomolecules in both systems are surrounded by an explicit solvent (water molecules and ions). Detailed methods used to generate the systems and the system construction are explained in Section 2 of SI.

\section{Three reaction coordinates}

We introduced three RCs to sample various conformations for two systems by GA-guided mD$\mathrm{VcMD}$. The basic method to define an RC was explained in section 1 of SI. Then, three $\mathrm{RCs} \lambda^{(\alpha)}$, $\lambda^{(\beta)}$, and $\lambda^{(\gamma)}$ were defined by the inter-mass-center distances between the two atom groups $G_{h}^{A}$ and $G_{h}^{B}(h=\alpha, \beta, \gamma)$, as presented in Figure 1. Details of the atom groups are given in Table S1 of section 4 of SI.

Here, we explain the RCs briefly: $\lambda^{(\beta)}$ represents the distance between Sep 34/Ser $34\left(=G_{\beta}^{B}\right)$ of pMLF1 or npMLF1 and a helical segment $\left(=G_{\beta}^{A}\right)$ of $14-3-3 \varepsilon$ near Sep 34/Ser 34 in the crystal structure. This distance controls the motion of Sep 34/Ser 34 and consequently, the salt-bridge formation between $14-3-3 \varepsilon$ and pMLF in the pMLF1/14-3-3 $\varepsilon$ system, whereas $G_{\beta}^{A}$ does not involve the counterpart of the salt bridge. For the pMLF1/14-3-3 $\varepsilon$ system, $\lambda^{(\beta)}$ simply controls the approach of npMLF1 to 14-3-3e. The RCs $\lambda^{(\alpha)}$ and $\lambda^{(\gamma)}$ control the motions of the N-terminal and C-termini of pMLF1 or npMLF1. In general, smaller RCs have closer conformation to the crystal structure. The RCs for the crystal structure are $\lambda^{(\alpha)}=15.6 \AA, \lambda^{(\beta)}=7.9 \AA$, and $\lambda^{(\gamma)}=8.7 \AA$. 
(A)

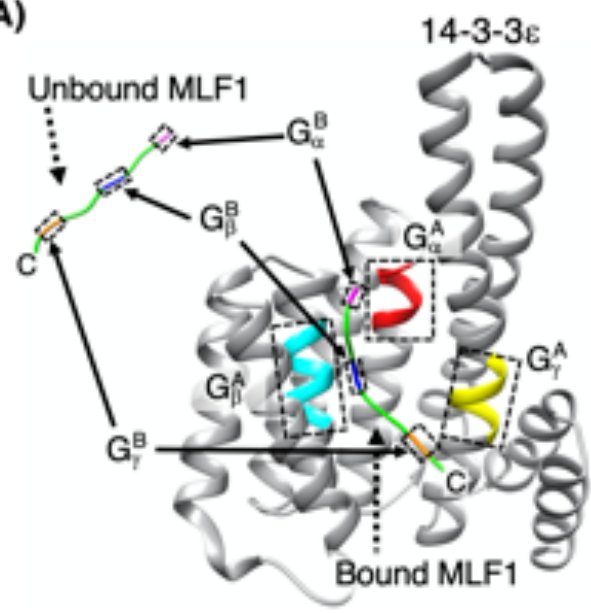

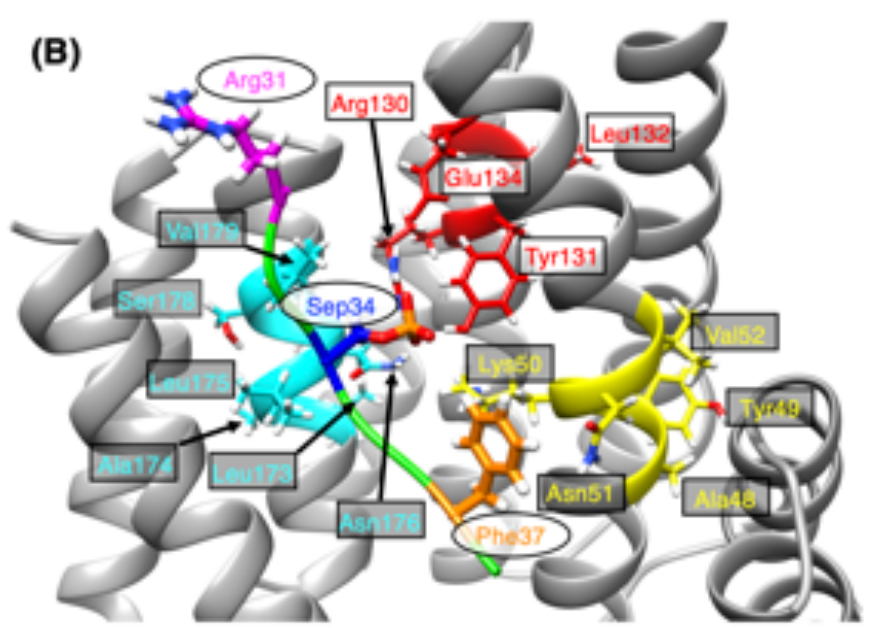

Figure 1. Atom groups to define three $\operatorname{RCs} \lambda^{(h)}(h=\alpha, \beta, \gamma)$ for pMLF1/14-3-3e. (A) Receptor 14-3-3 $\varepsilon$ and ligand pMLF1 are shown respectively in gray and green. Bound and unbound forms of pMLF1 are presented. $\lambda^{(\alpha)}$ is defined by the mass-center distance between two atom groups $G_{\alpha}^{A}$ (red fragment) and $G_{\alpha}^{B}$ (magenta), $\lambda^{(\beta)}$ by $G_{\beta}^{A}$ (cyan) and $G_{\beta}^{B}$ (blue), and $\lambda^{(\gamma)}$ by $G_{\gamma}^{A}$ (yellow) and $G_{\gamma}^{B}$ (golden yellow). Each atom group is surrounded by a broken-line rectangle. Section 1 of SI for definition of atom groups. Character "C" is assigned to the C-terminal of pMLF1. (B) Sidechains involved in the atom groups are shown explicitly

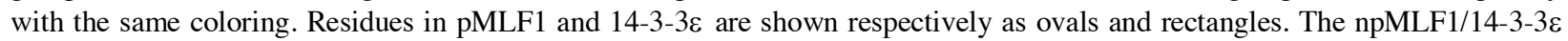
system is not shown. Table S1 of SI presents details of atom groups. Residues Ala 133, Asn 176, and Phe 177 of 14-3-3e are not shown because they are located at positions behind.

\section{Initial conformations of simulation}

The initial conformation of the GA-guided VcMD simulation is not the crystal complex structure but one for which pMLF1 (or npMLF1) is distant from the position in the native complex structure. The conformation of pMLF1 (or npMLF1) is randomized. We generated those conformations according to the method explained in section 3 of SI. Figure S5 presents additional details. We generated 256 initial conformations because we performed 256 runs of GA-guided VcMD as described below.

\section{Simulations}

Although the current system is a single heterodimer (Figure S4C), 14-3-3e maintained its tertiary structure in preliminary NVT simulations (data not shown). Therefore, no structural restraint was introduced to maintain the 14-3-3 $\varepsilon$ structure during the GA-guided $\mathrm{mD}-\mathrm{VcMD}$ simulations. To raise the sampling efficiency, we performed 256 runs in parallel ${ }^{4+12}$ for both system starting from randomized conformations generated in the section above and in section 3 of SI.

The GA-guided $\mathrm{mD}-\mathrm{VcMD}$ simulation was performed using a computer program, omegagene/myPresto ${ }^{43}$ with the following condition: The SHAKE algorithm ${ }^{4}$ is used to fix the covalent-bond lengths related to hydrogen atoms, the Berendsen thermostat to control temperature, ${ }^{45}$ the zero-dipole summation method ${ }^{4+48}$ for long-range electrostatic computations, time-step of $2 \mathrm{fs}$ ( $\Delta t=2 \mathrm{fs}$ ), and simulation temperature of $300 \mathrm{~K}$. An ensemble from the Berendsen thermostat can approximate a canonical distribution for a system of many atoms, whereas it generates a non-physical distribution for a small system. ${ }^{49}$ To compute the original potential energy of the system, the Amber hybrid force fields $s^{s \circ}$ (mixture parameter $w=0.75$ ) were used for pMLF1, npMLF1, and 14-3-3e, the TIP3P model for water molecule, , $^{\text {,1 }}$ and the Joung-Cheatham model for chloride and sodium ions..$^{22}$

\section{RESULTS}




\section{Conformational distribution in 3D-RC space}

We show in this section that the two systems provide greatly different conformational distributions from one another. The most stable state for pMLF1/14-3-3e involved crystal structure-like conformations. That for npMLF1/14-3-3e consists of conformations far from the crystal structure.

We performed GA-guided $\mathrm{mD}-\mathrm{VcMD}$ simulations and obtained an equilibrated conformational ensemble at $300 \mathrm{~K}$ for each system. The simulation condition is shown in section 4 of SI. We calculated a 3D density function, denoted as $\rho_{3 D-R C}(\lambda)=\rho_{3 D-R C}\left(\lambda^{(\alpha)}, \lambda^{(\beta)}, \lambda^{(\gamma)}\right)$, in the 3D-RC space from the obtained ensemble: The probability of detecting the system in a small RC volume $d \lambda=d \lambda^{(\alpha)} d \lambda^{(\beta)} d \lambda^{(\gamma)}$ is given as $\rho_{3 D-R C}(\lambda) d \lambda$. Figure 2 shows the resultant density functions

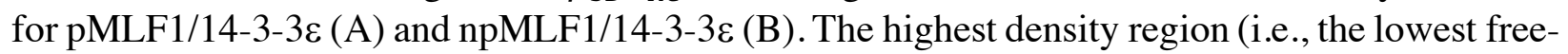
energy region or the most thermodynamically stable region) differed between the two systems. In pMLF1/14-3-3e, as shown later, the most stable region involved complex structures similar to the crystal structure. In npMLF1/14-3-3e, the most stable region consists of conformations where npMLF1 and 14-3-3e were unbound or were slightly contacting. Later, we demonstrate that this difference in the most stable region derives from the presence or absence of salt-bridges between MLF1 and 14-3-3e. Accuracy and convergence of distribution $\rho_{3 D-R C}(\lambda)$ for both systems are discussed in Figure S6 of SI.

Whereas the two systems provided different landscapes, two clusters existed in common. We designated them as $C_{1}$ and $C_{2}$ for pMLF1/14-3-3 $\varepsilon$ and $C_{1}^{\prime}$ and $C_{2}^{\prime}$ for npMLF1/14-3-3 $\varepsilon$ as in Figure 2. Clusters $C_{1}$ and $C_{2}$ correspond roughly to $C_{1}^{\prime}$ and $C_{2}^{\prime}$, respectively. In either system, the two clusters are connected by a corridor. Cluster $C_{1}$ is more stable than $C_{2}$, whereas $C_{1}^{\prime}$ is considerably less stable than $C_{2}^{\prime}$. Cluster $C_{1}^{\prime}$ exists marginally as a cluster.

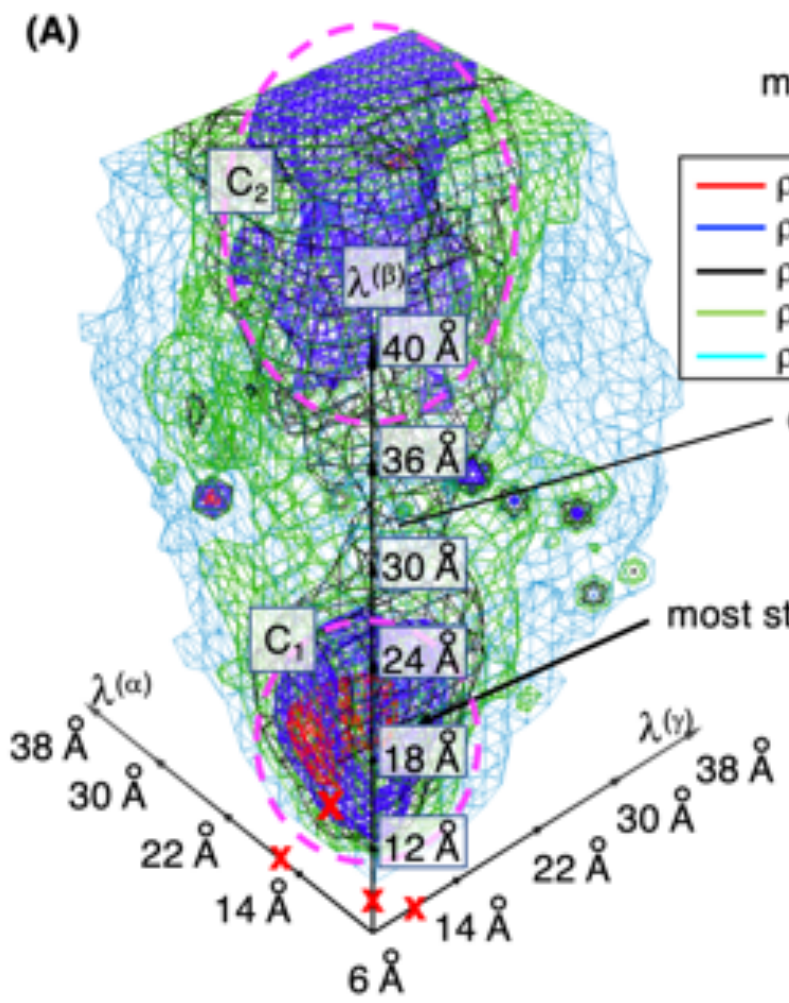

(B) most stable 
inset. The distribution is normalized as $\int \rho_{3 D-R C}\left(\lambda^{(\alpha)}, \lambda^{(\beta)}, \lambda^{(\gamma)}\right) d \lambda^{(\alpha)} d \lambda^{(\beta)} d \lambda^{(\gamma)}=1$. Clusters are shown by broken-line circles with labels $C_{1}, C_{2}, C_{1}^{\prime}$, and $C_{2}^{\prime}$. Corridors connecting $C_{1}$ and $C_{2}$ as well as $C_{1}^{\prime}$ and $C_{2}^{\prime}$ are indicated by "corridor". Positions of the most stable (highest density) regions are indicated by "most stable". The RCs for the crystal structure are indicated by red-colored crosses on their axes: $\lambda^{(\alpha)}=15.6 \AA, \lambda^{(\beta)}=7.9 \AA$, and $\lambda^{(\gamma)}=8.7 \AA$. Red-colored cross in the distribution indicates the crystalstructure position.

Here, to assess the conformational difference of pMLF1 (or npMLF1) between a snapshot and the crystal structure, we defined a measure $R M S D_{\text {lig }}$ : the mainchain heavy-atomic root mean square deviation for residues from Phe 33 to Phe 37 in pMLF1 (or npMLF1) after superposition of the 14-3-3e protein part between the snapshot and the crystal structure. Therefore, $R M S D_{\text {lig }}$ reflects not only the internal motions of pMLF1 (or npMLF1) but also its translational and rotational motions relative to 14-3-3e. As described in the Introduction, the contribution of aminoacids to the complex stability was measured for Phe 33, Glu 35, and Phe33. The most important residue for the complex formation is Sep 34.' Consequently, these amino-acids exist in residues 33-37. Residues Phe 33 and Phe 37 are located at the edges for the residue region.

Figures $3 \mathrm{~A}$ and 3B present conformations of the pMLF1/14-3-3e system taken from cluster $C_{1}$. Panel A displays the pMLF1 conformation with the smallest $R M S D_{\text {lig }}(0.69 \AA)$. The sidechain positions of Sep 34 and Phe 37 coincide well with those in the crystal structure shown in yellow. Although Phe 33 is deviated from the crystal-structure position, this residue interacts with Leu 230 via a hydrophobic contact, as discussed later. Panel $\mathrm{B}$ is a conformation picked randomly from cluster $C_{1}\left(R M S D_{\text {lig }}=4.0 \AA\right)$. Whereas Phe 33 and Phe 37 were deviated from the crystalstructure positions, the position of Sep 34 agreed well with the crystal-structure position. We present three conformations picked randomly from cluster $C_{2}$ in Figure S7A of SI, where pMLF1 was far from the crystal-structure position, and contacting to 14-3-3e (encounter complexes) or isolated in solution.

Figures 3C and 3D display conformations of the npMLF1/14-3-3e system from $C_{1}^{\prime}$. Panel $\mathrm{C}$ is the smallest $R M S D_{\text {lig }}$ conformation in cluster $C_{1}^{\prime}\left(R M S D_{\text {lig }}=1.60 \AA\right)$. Panel D presents a 
conformation chosen randomly from cluster $C_{1}^{\prime}\left(R M S D_{\text {lig }}=3.3 \AA\right)$. Three conformations selected randomly from cluster $C_{2}^{\prime}$ are shown in Figure S7B of SI.

Figure 3. Panels (A) and (B) display conformations from the pMLF1/14-3-3e system; (C) and (D) show those from the npMLF1/143-3e system. Yellow-colored MLF1 conformations in all panels are those from the crystal structure. Residues denoted by ovals
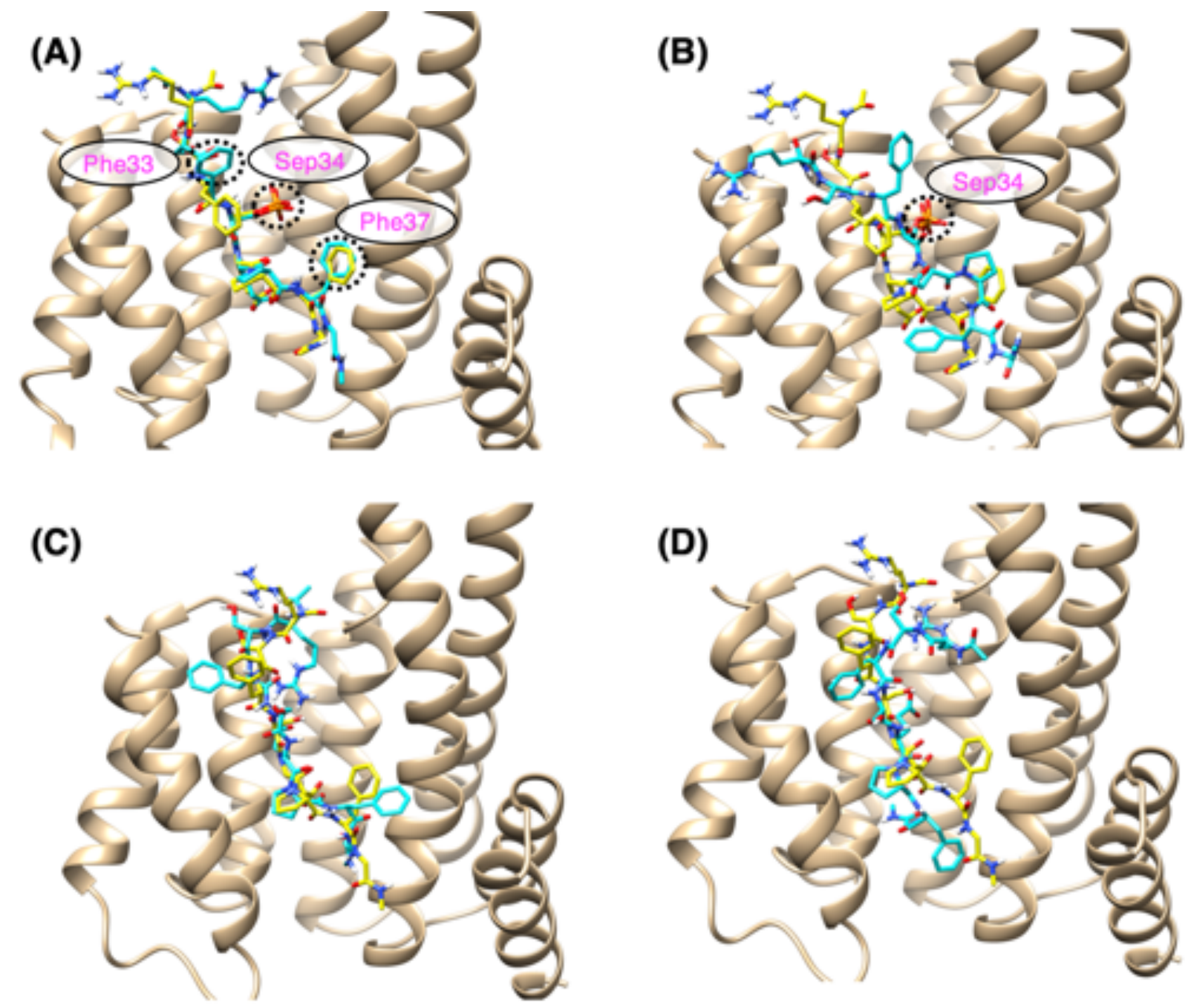

belong to pMLF1. The pMLF1 in (A) is the smallest $R M S D_{\text {lig }}$ conformation from cluster $C_{1}$. The pMLF1 in (B) is a randomly chosen conformation from cluster $C_{1}$. Residues Phe 33, Sep 34, and Phe 37 in (A) and Sep 34 in (B) are indicated by broken-line circles. Panel (C) is the smallest $R M S D_{\text {lig }}$ conformation from cluster $C_{1}^{\prime}$. Panel (D) is one randomly chosen conformation from cluster $C_{1}^{\prime}$.

\section{Distribution of MLF1 around $14-3-3 \varepsilon$ at $300 \mathrm{~K}$}

The earlier section showed that a single-residue difference (Sep/Ser) of MLF1 greatly varied the conformational distribution. Consequently, phosphorylation of MLF1 crucially governs the complex stability. This section presents further analysis of the difference of the complex stability between the two systems by calculating one-dimensional radial distribution functions.

As described in the Introduction, Molzan et al. reported that Phe 33 contributed greatly to complex-structure stabilization, in contrast to Glu 35 and Phe 37, which did not contribute substantially to the stability. Therefore, we specifically examined the following four inter-residue distances: The first distance $r^{\text {Phe } 33}$ is the minimum distance from the ring center of Phe 33 of pMLF1 (or npMLF1) to the sidechain heavy atoms of the hydrophobic interaction partners, Leu 
223 and Leu 230, of 14-3-3ع (Figure S2). The second distance $r^{\text {Sep34 }}$ (or $r^{\text {Ser34 }}$ ) is the minimum distance from the $\mathrm{O} \gamma$ atom of Sep 34 of pMLF1 (or Ser 34 of npMLF1) to the $C \zeta$ atoms of Arg 57 and Arg 130 of 14-3-3e. The positions of these three residues are presented in Figure S8 of SI. We did not select the phosphate atom for Sep 34 for the definition of $r^{\text {Sep34 }}$ because Ser 34 does not involve a phosphate atom. To compare behaviors of Sep 34 and Ser 34, the same atom should be selected. The third distance $r^{\text {Glu35 }}$ is the minimum distance from the $\mathrm{C} \gamma$ atoms of Glu 35 of pMLF1 (or npMLF1) to the $\mathrm{N} \zeta$ atoms of the salt-bridge partner, Lys 50 and Lys 123, of 14-3-3e. The fourth distance $r^{P h e 37}$ is the minimum distance from the ring center of Phe 37 of pMLF1 (or npMLF1) to the heavy atoms in the stem of Lys 50 of 14-3-3e. A lysine sidechain has a long hydrophobic stem.

We calculated the radial distribution function, $p\left(r^{\mu}\right)$ with respect to the four distances defined above ( $\mu=$ Phe33, Sep 34/Ser 34, Glu35, or Phe37). Details for the calculation of $p\left(r^{\mu}\right)$ are presented in section 5 of SI. Figure 4 depicts the calculated radial distribution functions. A remarkable difference between the two systems is related to Sep/Ser 34: the sharp peak of $p\left(r^{\text {Sep34 }}\right)$ is portrayed in Figure 4A, although $p\left(r^{\text {Ser34 }}\right)$ shows broad peaks (Figure 4B). This difference is attributable to the presence or absence of the salt bridges associated with Sep 34 and Ser 34, as discussed later. According to the decrease of the peak height from $p\left(r^{\text {Sep34 }}\right)$ to $p\left(r^{\text {Ser } 34}\right)$, the peaks of the other radial distribution functions decreased. This result derives naturally from Figure 3B: Cluster $C_{1}^{\prime}$ is less stable than $C_{2}^{\prime}$ in the npMLF1/14-3-3 $\varepsilon$ system. 


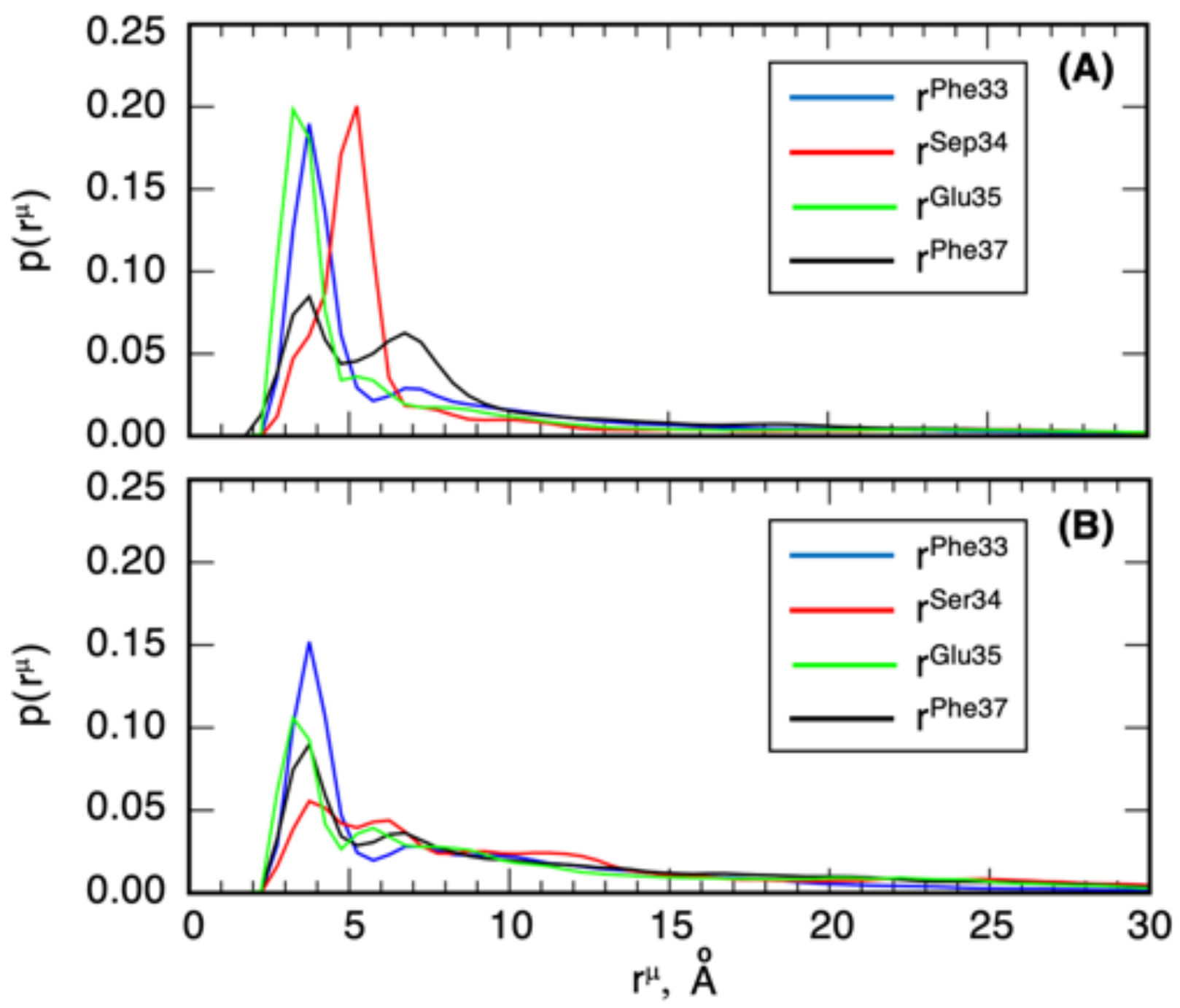

Figure 4. Radial distribution function $p\left(r^{\mu}\right)$ for four distances: $\mu=$ Phe33, Sep/Ser34, Glu35, and Phe37. See section 5 of SI for definition of $p\left(r^{\mu}\right)$. Panels (A) and (B) respectively show pMLF1/14-3-3e and npMLF1/14-3-3e.

Furthermore, in the pMLF1/14-3-3e system, it is noteworthy that $p\left(r^{\text {Phe33 }}\right)$ has a sharp peak, whereas the distribution of $p\left(r^{\text {Phe37 }}\right)$ is broad (Figure $\left.4 \mathrm{~A}\right)$. This result supports the experimental data showing that Phe 33 of pMLF1 contributes more to complex stability than Phe 37 of pMLF1 does.? Later, this point is discussed further.

The situation of Glu 35 of pMLF1 is problematic: $p\left(r^{G l u 35}\right)$ has a sharp peak at $r^{G l u 35} \approx 3 \AA$ in the pMLF1/14-3-3e system (Figure 4A). Consequently, Glu 35 seems to form a salt bridge to Lys 50 or Lys 123 of $14-3-3 \varepsilon$ in the simulation. This result might indicate that Glu 35 contributes to the complex stability. However, as described in the Introduction, the mutation E35A of pMLF1 did not change the complex stability. Later, we analyze fluctuations of Glu 35 around 14-3-3e to explain this discrepancy rationally.

One might note that most plots of Figure 4 had multiple peaks (or shoulders). The origin of this multiple-peak feature is the existence of multiple conformational clusters of MLF1 on the 14-3-3e surface, as shown later by 3D spatial distributions. 


\section{D spatial distribution of MLF1 around $14-3-3 \varepsilon$ at $300 \mathrm{~K}$}

We further investigate the 3D-spatial distribution (density map), $\rho_{\text {Sep } 34}^{O \gamma}(\boldsymbol{r})$ and $\rho_{\text {Ser } 34}^{O \gamma}(\boldsymbol{r})$, of the Sep 34 and Ser 34 positions around 14-3-3e to view the shape of the free-energy landscape for the positions of Sep 34 and Ser 34. The method used to compute $\rho_{\text {Sep34 }}^{o \gamma}(\boldsymbol{r})$ and $\rho_{\text {Ser } 34}^{O \gamma}(\boldsymbol{r})$ is given in section 6 of SI. In fact, the distribution can be converted to the potential mean force (PMF) simply as $P M F=-R T \ln \left[\rho_{\mu}^{o \gamma}(\boldsymbol{r})\right](\mu=\operatorname{Sep} 34$ or $\operatorname{Ser} 34)$.

Figure 5A shows density maps of $\rho_{\text {Sep } 34}^{O \gamma}(\boldsymbol{r})$, which is the spatial density of the O $\gamma$ atom of Sep 34 of pMLF1. Apparently, in the pMLF1/14-3-3e system, the high-density spot was found around the $\mathrm{O} \gamma$-atomic position of Sep 34 in the crystal structure (red contours in Figure 5A). This figure also shows that Sep 34 is distributed over almost the entire surface of 14-3-3e at a lower density level (dark green contours). However, at a middle density level (magenta-colored contours), the distribution was inhomogeneous with four large clusters $D_{1}, \ldots, D_{4}$. Cluster $D_{1}$ involved the highdensity spot described above. The other clusters did not involve such a high-density region. Consequently, $D_{1}$ was the most stable of the four clusters thermodynamically. Clusters $D_{1}$ and $D_{2}$ were connected smoothly without density discontinuity: In other words, $D_{1}$ and $D_{2}$ might be a single cluster. In contrast, density discontinuity (free-energy barriers) was found among clusters $D_{1}, D_{3}$, and $D_{4}$.

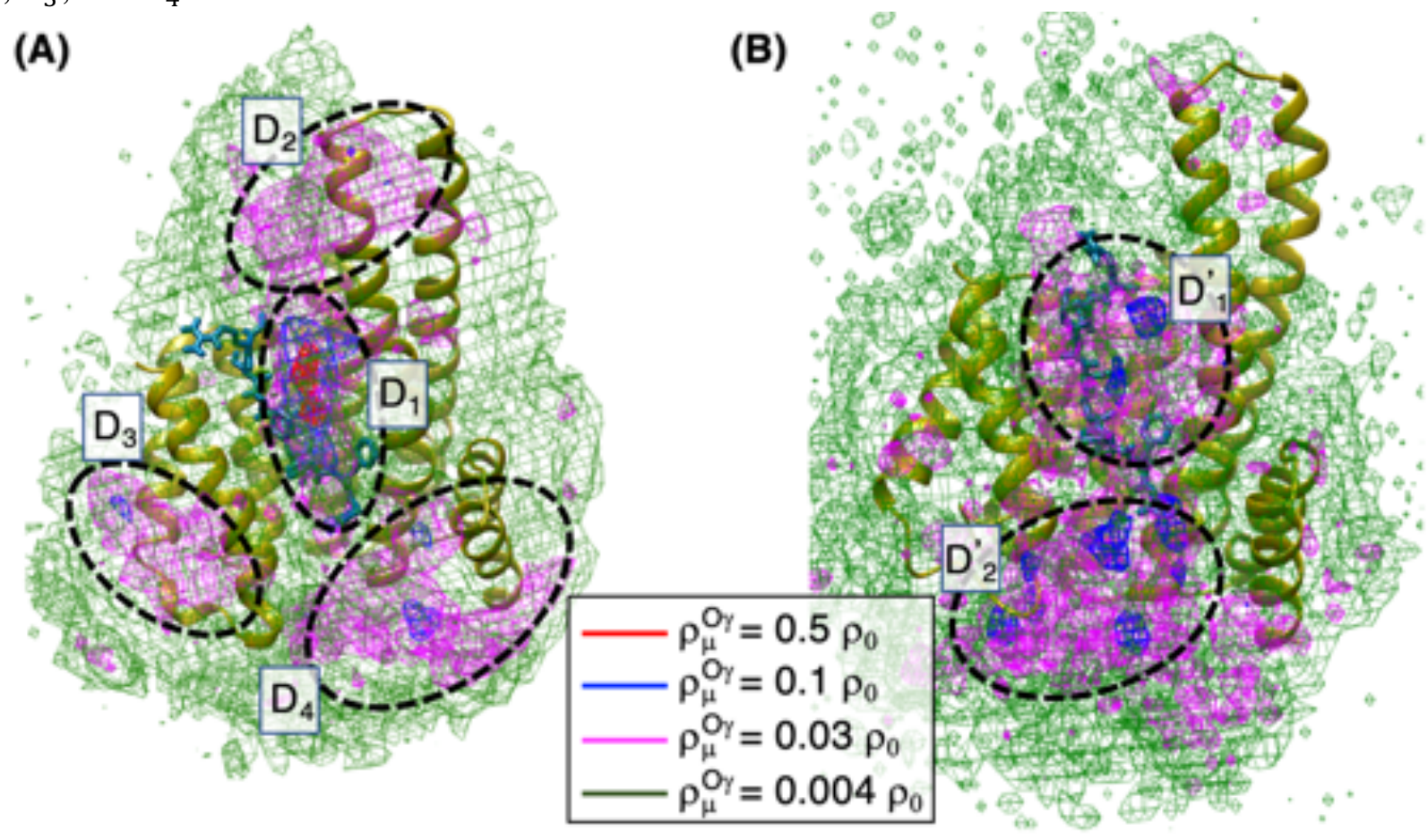

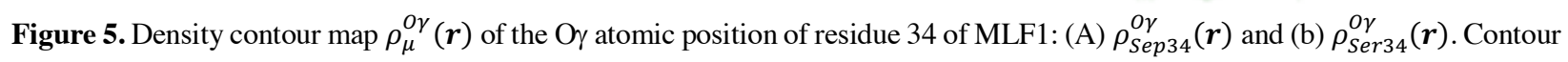
levels are shown in the inset, where $\rho_{0}=0.01$. The molecular structures shown are the crystal complex structure. Clusters surrounded by broken-line circles, designated as $D_{i}(i=1, \ldots, 4)$ and $D_{i}^{\prime}(i=1,2)$, are described in text.

Figure 5 shows that GA-guided $\mathrm{mD}-\mathrm{VcMD}$ can sample not only high-density regions but also low-density ones. Therefore, we presume that oversight of low-probability conformations did not occur. The minor-basin sampling might be fundamentally important to investigate connections among major basins, as described in the Introduction. 
To analyze cluster $C_{1}$ (Figure $2 \mathrm{~A}$ ), we calculated the density map of $\rho_{\text {Sep } 34}^{\text {or }}(\boldsymbol{r})$ using snapshots only from cluster $C_{1}$. Figure $\mathrm{S} 9$ of SI shows that conformations in $C_{1}$ involved cluster $D_{1}$ and a part of $D_{2}$ of Figure 5A. It is particularly interesting that clusters $D_{3}$ and $D_{4}$ are not found in Figure S9. Remember that clusters $D_{3}$ and $D_{4}$ are not interesting for discussion of binding pathways because free-energy barriers exist between $D_{3}$ and $D_{1}$ and between $D_{4}$ and $D_{1}$. However, the existence of $D_{3}$ and $D_{4}$ is important to characterize the free-energy landscape of this system: This landscape is not funnel-like but rather multi-modal, as found computationally before..$^{37.53}$

Figure $5 \mathrm{~B}$ is a contour map of $\rho_{\text {Ser } 34}^{O \gamma}(\boldsymbol{r})$ from the npMLF1/14-3-3e system. No red contours were found in this density map, which results from absence of a salt bridge at the 34 th residue. We found two clusters, denoted as $D_{1}^{\prime}$ and $D_{2}^{\prime}$, in Figure $5 \mathrm{~B}$ at the level of $\rho_{\operatorname{Ser} 34}^{O \gamma}(\boldsymbol{r})=0.03 \rho_{0}$ (magenta-colored contours). The position of $D_{1}^{\prime}$ overlaps that of $D_{1}$ in Figure $5 \mathrm{~A}$, revealing that npMLF1 has a weak tendency of staying around the pMLF1 position in the crystal structure.

\section{Salt-bridge relay between pMLF1 and 14-3-3ع}

As presented in Figure $5 \mathrm{~A}$, cluster $D_{1}$ was the most stable of the four clusters. Here, we investigate the internal structure of cluster $D_{1}$. Figure $6 \mathrm{~A}$ shows that the contours of $\rho_{\text {Sep } 34}^{O \gamma}=$ $0.5 \rho_{0}$ are split into two spots, named $E_{1}$ and $E_{2}$, and that $E_{1}$ involves the crystal-structure position of the Oy atom of Sep 34 of pMLF1.

As described above, a probable binding pathway is that from cluster $D_{2}$ to $D_{1}$ because no freeenergy barrier is apparent between $D_{1}$ and $D_{2}$. Therefore, the conformational change of pMLF1 from $D_{2}$ to $D_{1}$ is noteworthy. Figure $6 \mathrm{~B}$ is a snapshot taken from a fringe of $E_{2}$, where Sep 34 formed a salt bridge to Arg 61 of 14-3-3e, which is not observed in the crystal structure. Figure 6C displays a snapshot taken from the middle of $E_{2}$, where Sep 34 formed salt bridges to Arg 61 and Arg 57 of 14-3-3e. The salt bridge to Arg 57 is one observed in the crystal structure. Additionally, it is noteworthy that Arg 130 of 14-3-3e did not participate in the salt bridge to Sep 34. Figure 6D portrays a snapshot selected from the corridor between $E_{1}$ and $E_{2}$, which shows that the salt bridge to Arg 61 was broken and that the salt bridge to Arg 130 was not formed. Consequently, the conformation at the corridor had only one salt bridge, which suggests that the conformation at the corridor is energetically less stable than that in $E_{2}$. Figure $6 \mathrm{E}$ presents a snapshot taken from $E_{1}$, in which the two salt bridges were formed as in the crystal structure. We presume that the corridor between $E_{1}$ and $E_{2}$ originated from decrease of a salt bridge. Consequently, Figures 6B-6E suggest that the salt-bridge relay takes place when the conformation moves from $E_{2}$ to $E_{1}$. 


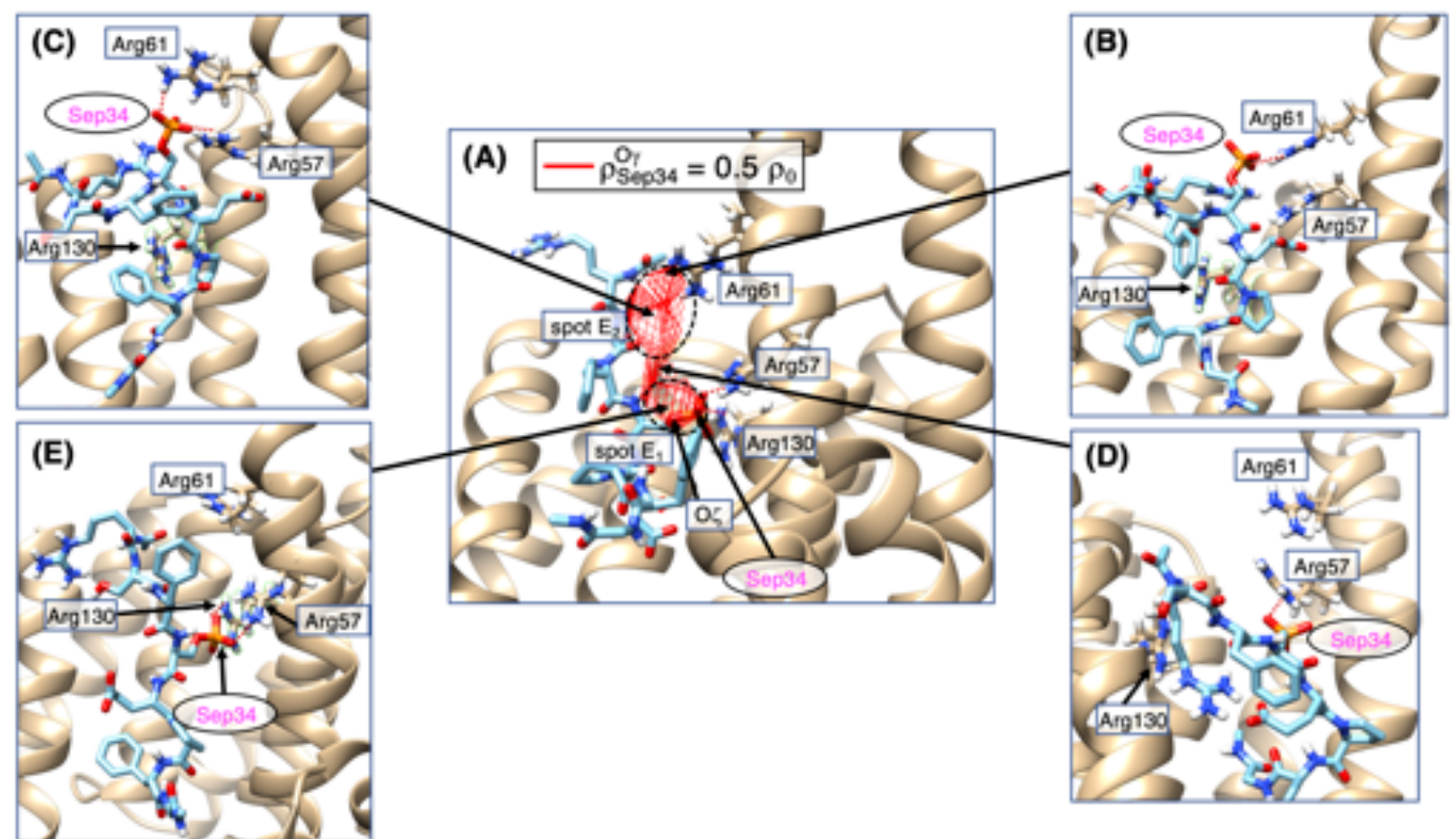

Figure 6. (A) Close-up of cluster $D_{1}$. Red-colored contours are those with $\rho_{\text {Sep } 34}^{o \gamma}=0.5 \rho_{0}\left(\rho_{0}=0.01\right)$. Two high-density spots $E_{1}$ and $E_{2}$ are shown. (B) Snapshot taken from the fringe of $E_{2}$. (C) Snapshot taken from the middle of $E_{2}$. (D) Snapshot taken from the corridor connecting $E_{1}$ and $E_{2}$. (E) Snapshot taken from $E_{1}$. The salt bridge is shown as a brown broken line. Residues labeled in ovals belong to pMLF1, and those in rectangles do to 14-3-3e.

\section{Spatial distribution of Phe 33 and Phe 37 of MKF1 around 14-3-3e at $300 \mathrm{~K}$}

As described in the Introduction, Phe 33 of pMLF1 contributes greatly to the complex stability, whereas Phe 37 does not. Next, we investigate the spatial distributions of Phe 33 and Phe 37 of pMLF1 around 14-3-3e to elucidate the contributions of these residues to the complex stability.

Figure S2 displays the pMLF1/14-3-3e complex structure (crystal structure), where Phe 33 of pMLF1 is sandwiched between Leu 223 and Leu 230 of 14-3-3e, and Phe 37 of pMLF1 contacts to the hydrophobic sidechain stem of Lys 50 of 14-3-3e. We calculated the density of the sidechain ring centers of Phe 33 and Phe 37, which are expressed respectively by $\rho_{\text {Phe33 }}^{\text {ring }}(\boldsymbol{r})$ and $\rho_{\text {Phe } 37}^{\text {ring }}(\boldsymbol{r})$ of pMLF1 (Figure 7A) or npMLF1 (Figure 7B). Section 6 of SI presents the procedure used to calculate the densities. For the pMLF1/14-3-3 $\varepsilon$ system, Phe 33 distributed in a region named spot $H$ in Figure 7A, which is capable of contacting Leu 223 and/or Leu 230. Spot $H$ is the largest spot

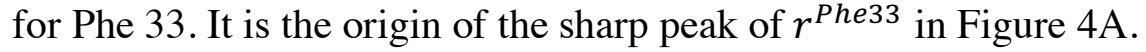

Two spots $F_{1}$ and $F_{2}$ for $\rho_{P h e 37}^{\text {ring }}(\boldsymbol{r})$ in Figure 7A are related to the two peaks of $p\left(r^{\text {Phe37 }}\right)$ in Figure $4 \mathrm{~A}$. That is, $F_{1}$ and $F_{2}$ respectively represent the origins of the peaks at $r^{\text {Phe37 }} \approx 4 \AA$ and $r^{\text {Phe37 }} \approx 7 \AA$. In contrast to the large spot $H$ for Phe 33, both spots $F_{1}$ and $F_{2}$ for Phe 37 were small, which suggests that Phe 37 contributes less to stabilization of the complex structure.

In Figure 7B, the red and blue contours distributed widely and sparsely around 14-3-3e. It is noteworthy that Ser 34 in npMLF1/14-3-3e distributed in clusters $D_{1}^{\prime}$ and $D_{2}^{\prime}$ in Figure 5B. Figures 5B and 7B show that nPMLF1 has no strong preferential spot. Although the three broken-line circles, named as spots $H_{1}^{\prime}, H_{2}^{\prime}$, and $H_{3}^{\prime}$ in Figure 7B, were found to be close to Leu 223 and Leu 230, these spots were small. They caused the peak of $r^{P h e 33}$ in Figure 4B. 
We calculated the 3D distributions $\rho_{P h e 33}^{\text {ring }}(\boldsymbol{r})$ and $\rho_{P h e 37}^{\text {ring }}(\boldsymbol{r})$ for the pMLF1/14-3-3e system using snapshots only from cluster $C_{1}$ in Figure $2 \mathrm{~A}$. They are portrayed in Figure S10 of SI. Figure is similar to Figure 7A: $\rho_{P h e 33}^{\text {ring }}(\boldsymbol{r})$ is localized around the position of Phe 33 in the crystal structure; also, $\rho_{P h e 37}^{\text {ring }}(\boldsymbol{r})$ distributed broadly on the surface of 14-3-3 $\varepsilon$. However, the density level of Figure S10 was higher than that of Figure 7A for both Phe 33 and Phe 37 because the distribution was normalized only using snapshots in $C_{1}$ in Figure $\mathrm{S} 10$.

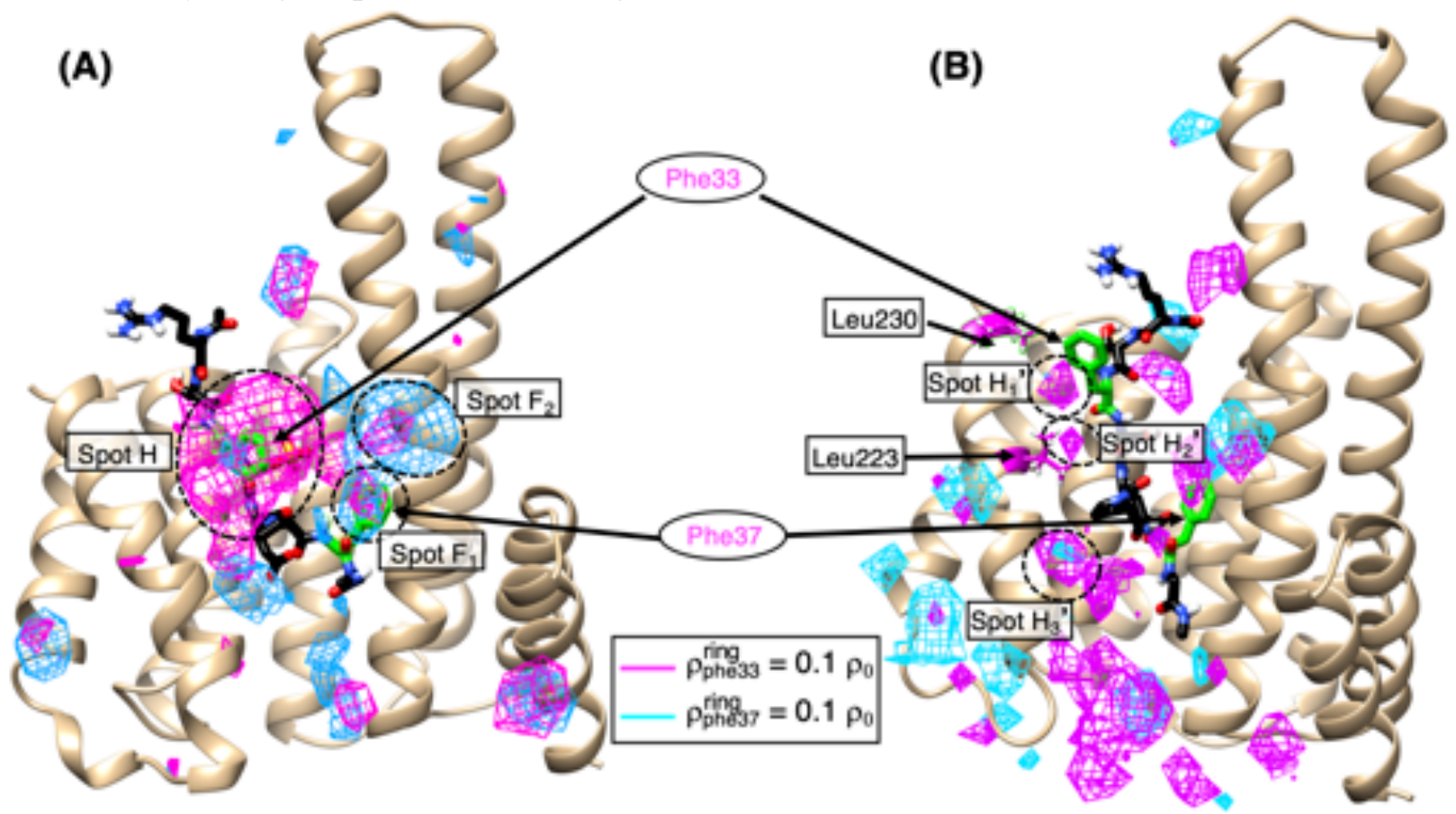

Figure 7. Spatial distributions of the sidechain ring-center positions of Phe 33 and Phe 37, denoted respectively as $\rho_{\text {Phe } 33}^{\text {ring (magenta- }}$ colored contours) and $\rho_{\text {Phe37 }}^{\text {ring }}$ (cyan-colored contours). The contour level is $0.1 \rho_{0}\left(\rho_{0}=0.01\right)$ for both residues. Panels (A) and

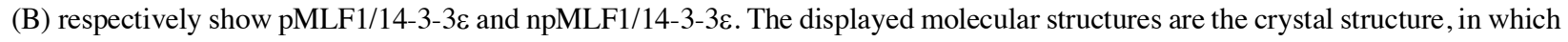
green residues are Phe 33 and Phe 37. Residues labeled in ovals and rectangles respectively belong to MLF1 and 14-3-3e. In panel (A), broken-line circles named spots $H, F_{1}$, and $F_{2}$ are described in the text. In panel (B), Leu 223 and Leu 230 are shown in magenta color. Three broken-line circles, named spots $H_{1}^{\prime}, H_{2}^{\prime}$, and $H_{3}^{\prime}$, are described in the text.

\section{Spatial distribution of Glu 35.}

In the crystal structure, the sidechain of Glu 35 of pMLF1 forms salt-bridges to Lys 50 and Lys 123 of 14-3-3e. However, as described in the Introduction, Glu 35 of pMLF1 does not contribute to the complex stability. To explain this discrepancy, we calculated the density maps $\rho_{G l u 35}^{C \alpha}(\boldsymbol{r})$ and $\rho_{G l u 35}^{C \gamma}(\boldsymbol{r})$, respectively, for the $\mathrm{C} \alpha$ and $\mathrm{C} \gamma$ atomic positions of Glu 35 of pMLF1 for the pMLF1/14-3-3e system. Section 6 of SI explains the procedure used to calculate the densities.

Figure 8A shows high-density regions drawn around the crystal structure, in which Glu 35 forms salt bridges to Lys 50 and Lys 123 of 14-3-3e. The contours show that both the $\mathrm{C} \alpha$ and $\mathrm{C} \gamma$ atoms shifted from the crystal-structure positions toward the solvent, and that the mainchain of Glu 35 fluctuated to a considerable degree, as represented by the broken-line arrow along the magentacolored contours in Figure 8A. We consider that this positional shift hinders Glu 35 of pMLF1 to form salt bridges to Lys 50 and Lys 123 of 14-3-3e. The black contours in Figure S11A demonstrate that Glu 35 can take a position (broken-line circle of Figure 11A), from which Glu 35 was able to form the salt bridges to both Lys 50 and Lys 123. However, the density assigned to 
this position was small $\left(\rho_{\text {Glu35 }}^{C \gamma}(\boldsymbol{r})=0.05 \rho_{0}\right)$. Consequently, it seems that Figure 8A and Figure $4 \mathrm{~A}$ are mutually contradictory.

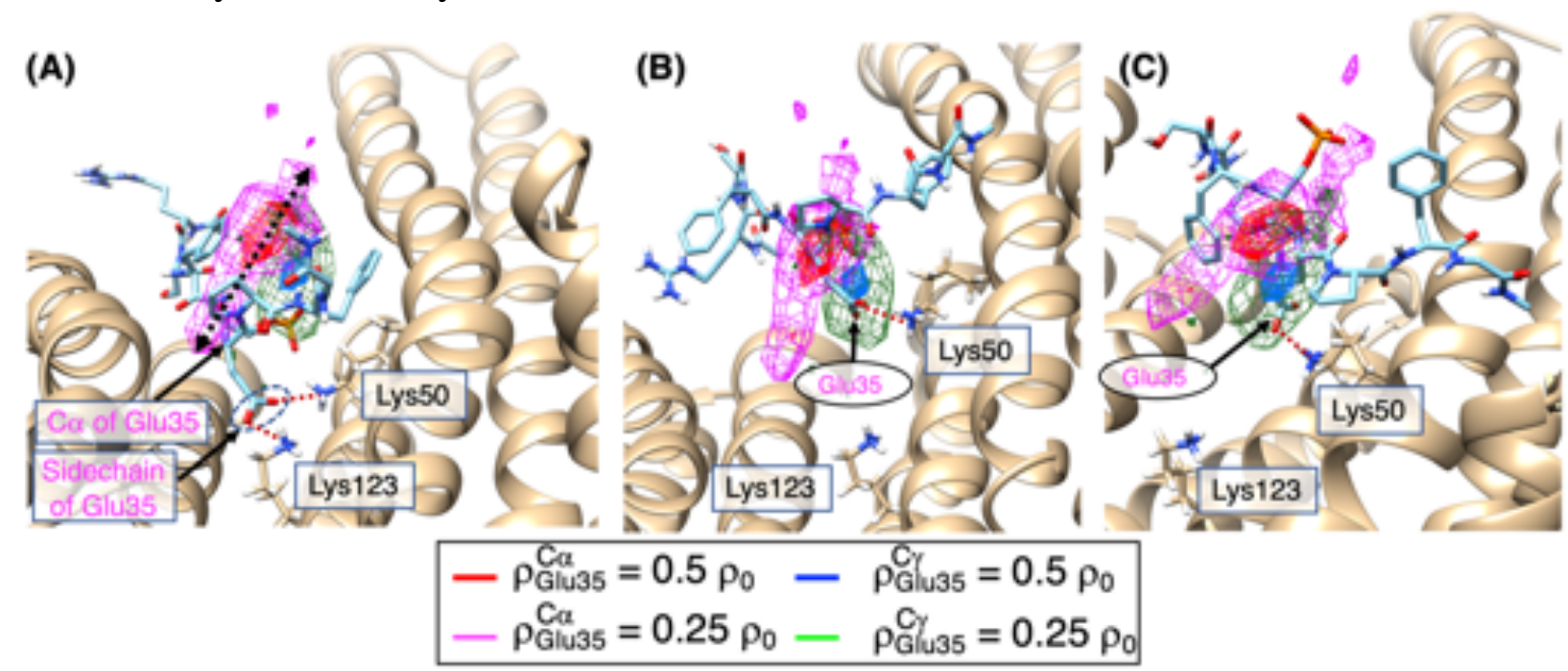

Figure 8. (A) Density contour maps of $\rho_{G l u 35}^{C \alpha}(\boldsymbol{r})$ and $\rho_{G l u 35}^{C \gamma}(\boldsymbol{r})$ for the pMLF1/14-3-3e system. The presented molecular structure is the crystal one, where the C $\alpha$ atom and sidechain tip of Glu 35 of pMLF1 are denoted respectively as "C $\alpha$ of Glu35" and "Sidechain of Glu35". Contour levels are shown in the inset $\left(\rho_{0}=0.01\right)$. Panels (B) and (C) display snapshots chosen randomly from conformations that are involved in the contour regions of panel (A). Panels (B) and (C) are drawn with different orientations from panel (A) to show Glu 35 clearly. Salt bridge is shown by the brown broken line. Residues labeled in ovals belong to pMLF1. Those in rectangles belong to $14-3-3 \varepsilon$.

To elucidate this discrepancy, we checked snapshots for which $\mathrm{C} \alpha$ and $\mathrm{C} \gamma$ atoms were involved in the contours of $\rho_{G l u 35}^{C \alpha}(\boldsymbol{r})=0.25 \rho_{0}$ (magenta contours in Figure 8A) and $\rho_{G l u 35}^{C \gamma}(\boldsymbol{r})=0.25 \rho_{0}$ (green contours of Figure 8A), and found that most of those snapshots in the contour regions formed a salt bridge only to Lys 50 of 14-3-3e. Figures $8 \mathrm{~B}$ and $8 \mathrm{C}$ present two snapshots selected randomly from the contour regions. To clarify this observation, we calculated $p\left(r^{G l u 35-L y s 50}\right)$ and $p\left(r^{G l u 35-L y s 123}\right)$ : The former is a radial distribution function for the distance $r^{G l u 35-L y s 50}$ from the $\mathrm{C} \gamma$ atom of Glu 35 of pMLF1 to the $\mathrm{N} \zeta$ atom of Lys 50 of 14-3-3e. The latter is a radial distribution function for the distance $r^{G l u 35-L y s 123}$ from the $\mathrm{C} \gamma$ atom of Glu 35 to the $\mathrm{N} \zeta$ atom of Lys 123 of 14-3-3e. These distances were used to define $r^{G l u 35}$ in Figure 4. Figure S11B clarifies that the salt bridge between Glu 35 and Lys 123 was rarely formed. Furthermore, we calculated salt-bridge-formation probabilities $Q_{G l u 35-L y s 50}$ and $Q_{G l u 35-L y s 123}$ according to section 7 of SI. The resultant fraction $Q_{G l u 35-L y s 123} / Q_{G l u 35-L y s 50}$ was $0.18 \times 10^{-5}$, which again indicated that the salt bridge between Glu 35 and Lys 50 was dominant.

In fact, Lys 50 of $14-3-3 \varepsilon$ is located on the surface of 14-3-3e. Consequently, the sidechain stem of Lys 50 can modulate its sidechain-tip position to maintain the salt bridge to Glu 35 of pMLF1. In contrast, Lys 123 is somewhat buried in 14-3-3e. Therefore, the sidechain tip of Lys 123 cannot move together with the positional shift of Glu 35. Consequently, the salt bridge between Glu 35 of pMLF1 and Lys 123 of $14-3-3 \varepsilon$ is not formed.

We presume that the positional shift and large fluctuations of Glu 35 of pMLF1 weaken the contribution of Glu 35 to the complex-structure stability. It is noteworthy that the sidechain stem of Lys 50 of $14-3-3 \varepsilon$ is the hydrophobic interaction partner of Phe 37 in the crystal structure. Therefore, the positional fluctuations of Glu 35 of pMLF1 affect the sidechain motions of Lys 50 of 14-3-3e. They therefore hinder the hydrophobic interaction between Phe 37 of pMLF1 and Lys 
50 of $14-3-3 \varepsilon$ : the small contributions of Glu 35 and Phe 37 to the complex structure stability might be mutually correlated.

\section{Orientation and ligand conformational variety of pMLF1 around 14-3-3e}

This section presents specific examination of the molecular orientation and the conformational variety of pMLF1 around $14-3-3 \varepsilon$ to elucidate the conformational convergence of pMLF1 with approach of the binding site of 14-3-3e. First, we defined the orientation of pMLF1 by a unit vector $\boldsymbol{e}_{i}\left(\left|\boldsymbol{e}_{i}\right|=1\right)$ for snapshot $i$, which is pointing from the C $\alpha$ atom of Phe 33 to the C $\alpha$ atom of Phe 37 of the snapshot. The orientation vector $\boldsymbol{e}_{\text {ref }}\left(\left|\boldsymbol{e}_{\text {ref }}\right|=1\right)$ for the crystal structure was also calculated similarly.

The ligand pMLF1, for which $\mathrm{O} \gamma$ atom of Sep 34 was visiting a cube $\boldsymbol{r}$, has an orientational variety. We calculated $\overline{\boldsymbol{v}}(\boldsymbol{r})$, the average of the orientation vectors $\boldsymbol{e}_{i}$ in a cube $\boldsymbol{r}$, using a method explained in section 8 of SI (Eq. S1). Note that the vector $|\overline{\boldsymbol{v}}(\boldsymbol{r})|$ shows a degree of orientational ordering of pMLF1 detected in cube $\boldsymbol{r}$; also, $|\overline{\boldsymbol{v}}(\boldsymbol{r})|$ takes the maximum of 1 when all $\boldsymbol{e}_{i}$ have exactly the same orientations in the cube. If snapshots have uncorrelated orientations, then $|\bar{v}(\boldsymbol{r})|$ becomes small.

Next, to elucidate the tendency of $\overline{\boldsymbol{v}}(\boldsymbol{r})$ to align to $\boldsymbol{e}_{\text {ref }}$ for the crystal structure or not, we calculated a scalar product of $\overline{\boldsymbol{v}}(\boldsymbol{r})$ and $\boldsymbol{e}_{r e f}$, and assigned it to cube $\boldsymbol{r}$ as

$$
S C(\boldsymbol{r})=\overline{\boldsymbol{v}}(\boldsymbol{r}) \cdot \boldsymbol{e}_{\text {ref }}
$$

which takes a value of -1 to 1 . Green and orange arrows in Figure 9A are vectors $\overline{\boldsymbol{v}}(\boldsymbol{r})$ that satisfy the following two conditions:

and

$$
\rho_{\text {Sep34 }}^{O \gamma}(\boldsymbol{r}) \geq 0.03 \rho_{0}
$$

$$
|\overline{\boldsymbol{v}}(\boldsymbol{r})| \geq 0.5
$$

Equation 2 is used to pick cubes involved in high-density regions (clusters $D_{1}, \ldots, D_{4}$ in Figure 5). Also, Eq. 3 is used to display well ordered $\overline{\boldsymbol{v}}(\boldsymbol{r})$. Apparently, only cluster $D_{1}$ exhibited remarkable orientational ordering of pMLF1. Its majority was occupied by $S C(\boldsymbol{r}) \geq 0.5$ (green arrows). In contrast, cubes with $S C(\boldsymbol{r})<0.5$ were rare and sparse. We infer that the orientation of pMLF1 was ordered only in cluster $D_{1}$ : In other words, the pMLF1 orientation is ordered as reaching the high-density region (i.e., low free-energy region) to be beneficial for binding to $14-3-3 \varepsilon$. 

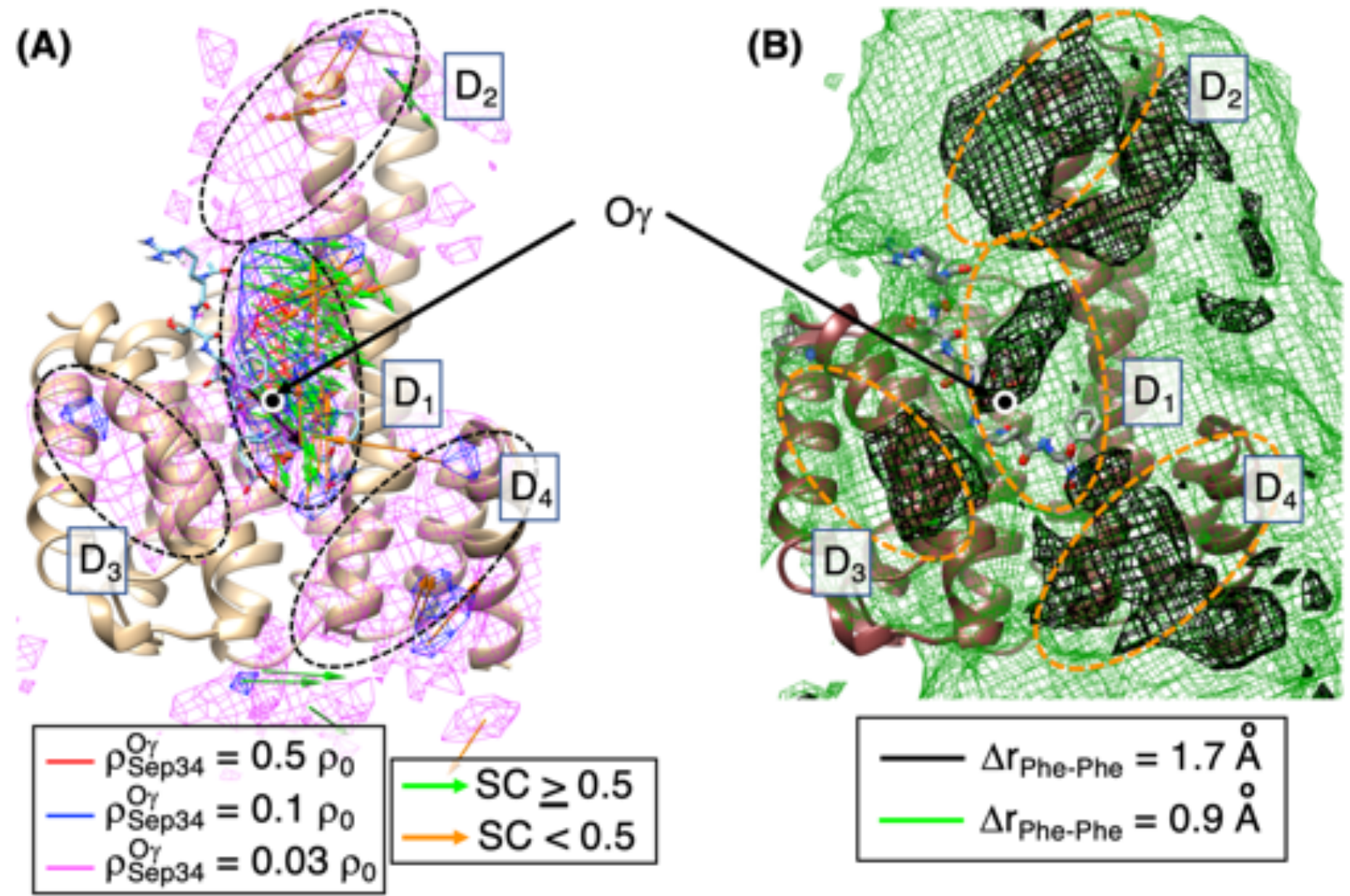

Figure 9. In both panels, the structure which is shown is crustal structure of pMLF1/14-3-3e, and positions of clusters $D_{1}, \ldots, D_{4}$, which were introduced in Figure 5A, are shown as broken-line circles. Positions of O $\gamma$ atom of Sep 34 of pMLF1 are shown by filled circles with label "O $\gamma$ ". (A) A veraged vectors $\overline{\boldsymbol{v}}(\boldsymbol{r})$ in cubes $\boldsymbol{r}$ satisfy Eqs. 2 and 3. Red-, blue-, and magenta-colored contours are the same as those in Figure 5A, although 14-3-3e is viewed in different orientation from Figure 5 to show the positiondependency of $\overline{\boldsymbol{v}}(\boldsymbol{r})$. Green and orange arrows respectively represent vectors with $S C(\boldsymbol{r}) \geq 0.5$ and $S C(\boldsymbol{r})<0.5$. Vectors are multiplied by 10.0 to show the vectors clearly: arrow $=10.0 \times \overline{\boldsymbol{v}}(\boldsymbol{r})$. Black arrow points from the $\mathrm{C} \alpha$ atom of Phe 33 to the $\mathrm{C} \alpha$ atom of Phe 37, which shows orientation of pMLF1 in the crystal structure, $\boldsymbol{e}_{r e f}$. (B) Spatial patterns of $\Delta r_{P h e-P h e}(\boldsymbol{r})$. Black contours show regions of $\Delta r_{P h e-P h e}(\boldsymbol{r})=1.7 \AA$. Green ones show regions of $0.9 \AA$.

Next, we calculated a ligand-conformational variety, $\Delta r_{P h e-P h e}(\boldsymbol{r})$, of pMLF1 at each cube $\boldsymbol{r}$. Details for $\Delta r_{P h e-P h e}(\boldsymbol{r})$ are presented in section 9 of SI. Larger $\Delta r_{P h e-P h e}(\boldsymbol{r})$ in a cube $\boldsymbol{r}$ are associated with a larger the ligand-conformational variety in the cube. We note that $\Delta r_{P h e-P h e}(\boldsymbol{r})$ is not linked directly to dynamic chain flexibility of pMLF1 because the conformational ensemble from the GA-guided $\mathrm{mD}-\mathrm{VcMD}$ is an equilibrated one at $300 \mathrm{~K}$.

Figure 9B demonstrates the spatial patterns of $\Delta r_{P h e-P h e}(\boldsymbol{r})$ at two contour levels: $\Delta r_{P h e-P h e}=$

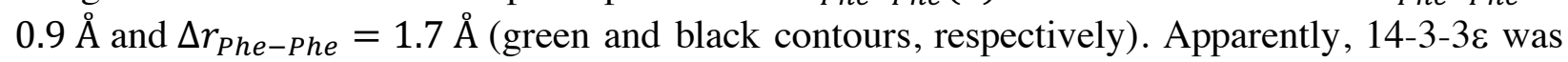
surrounded widely by the featureless contours of $\Delta r_{P h e-P h e}=0.9 \AA$. By contrast, the regions of $\Delta r_{P h e-P h e}=1.7 \AA$ were localized. Figure S12 presents the regions of $\Delta r_{P h e-P h e}=1.7 \AA$ as well as the density maps of $\rho_{\text {Sep } 34}^{O \gamma}=0.03 \rho_{0}$ from two orientations. Apparently, clusters $D_{2}, D_{3}$ and $D_{4}$ overlapped the regions of $\Delta r_{P h e-P h e}=1.7 \AA$ well. It is likely that a cube occupied by randomized pMLF1 conformations is characterized by less orientation ordering and a wide ligandconformational variety.

However, the situation of cluster $D_{1}$ differs from those of $D_{2}, D_{3}$ and $D_{4}$. As shown in Figure $9 \mathrm{~A}$, cluster $D_{1}$ was characterized by a well-ordered orientation. Figure $11 \mathrm{~B}$ demonstrates that the 
contours of $\Delta r_{P h e-P h e}=1.7 \AA$ overlapped with only a part of cluster $D_{1}$, and that the high-density spots $E_{1}$ and $E_{2}$, which were characterized by the higher contour level $\left(\rho_{\text {Sep } 34}^{O \gamma}=0.5 \rho_{0}\right)$ in Figure $6 \mathrm{~A}$, were not involved in the $\Delta r_{P h e-P h e}=1.7 \AA$ contours. The $O \gamma$ atom of Sep 34 in the crystal structure is involved in spot $E_{1}$. Therefore, we concluded that cluster $D_{1}$, to which the well-ordered ligand orientations were assigned, is not characterized by the large ligand-conformational variety.

\section{Site-dependence of residue interactions between pMLF1 and 14-3-3e}

The four distances $r^{\mu}(\mu=$ Phe33,Sep34,Glu35,Phe37) were defined for each snapshot for Figure 4. Investigating spatial patterns of these distances around $14-3-3 \varepsilon$ is interesting. For this purpose, we calculated thermal average $\bar{r}^{\mu}(\boldsymbol{r})$ of these distances for each cube $\boldsymbol{r}$ for the pMLF1/14-3-3e system according to the procedure explained in section 10 of SI. The thermal average $\bar{r}^{\mu}$ assigned to cube $\boldsymbol{r}$ is computed using snapshots for which the $\mathrm{O} \gamma$ atom of Sep 34 is detected in cube $\boldsymbol{r}$.

Figure 10A displays the overall spatial patterns of $\bar{r}^{\mu}(\boldsymbol{r})$ around 14-3-3e. The red contours show regions with $\bar{r}^{\operatorname{Sep34}}(\boldsymbol{r}) \leq 5.5 \AA$. One might consider that the $5.5 \AA$ value is larger than the limit distance for a salt bridge. However, the contours are assigned to the Or atom of Sep 34, not to three $\mathrm{O}$ atoms of the phosphate group. Therefore, we increased the limit distance. We also set $\bar{r}^{\text {Glu35 }}(\boldsymbol{r}) \leq 5.5 \AA$ as described before. The limits for Phe 33 and Phe 37 of pMLF1 were set to 7 $\AA: \bar{r}^{\mu}(\boldsymbol{r}) \leq 7 \AA$, which is larger than the value for a hydrophobic contact because $r^{\mu}(i)$ was calculated using the ring center (not individual atoms of the ring) of Phe 33 and Phe 37.

Contours for Phe 33 of pMLF1 (blue contour) occupied large regions in Figure 10A, which indicates that Phe 33 can form hydrophobic contacts to Leu 223 and Leu 230 of 14-3-3e readily. When the Oy atom of Sep 34 of pMLF1 was inside of the blue contour regions, Phe 33 was able to form the hydrophobic contact to its interaction partners of 14-3-3e. This result raises the possibility that these hydrophobic contacts might act as attractive interactions between pMLF1 and 14-3-3e. Although we have no definite answer to this argument, results of our earlier work examining $\mathrm{mD}-\mathrm{VcMD}^{39}$ suggest that a long $\mathrm{N}$-terminal tail of a protein might capture its ligand before the ligand binds to the binding site of the receptor (fly casting mechanism) ${ }^{54-57}$ Consequently, the hydrophobic contacts with respect to Phe 33 of pMLF1 might play a role in engendering the most stable complex structure.

(A)

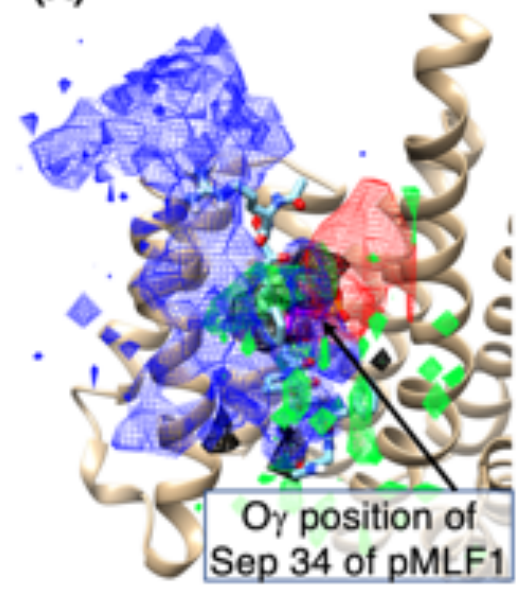

(B)

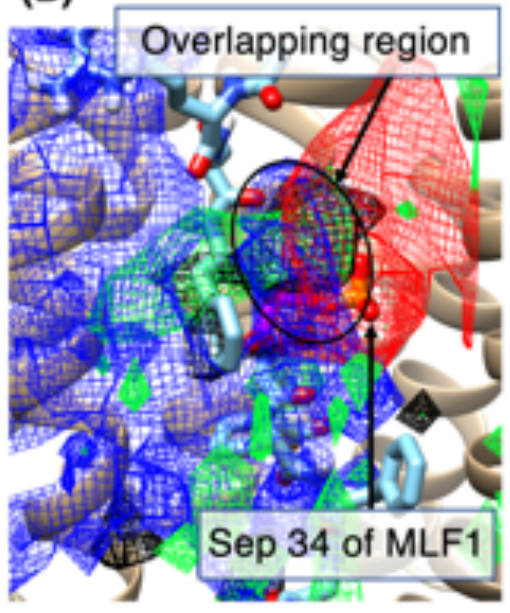

(C)

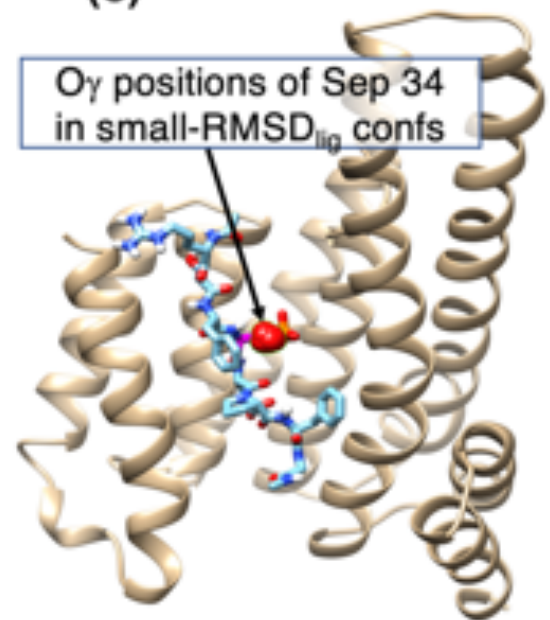

Figure 10. (A) Regions that satisfy $\bar{r}^{\mu} \leq r_{\text {lim }}$. Red-colored contour regions are those for $\mu=S e p 34$ of pMLF1 and $r_{l i m}=5.5 \AA$. Blue-colored contour regions are those for $\mu=P h e 33$ of pMLF1 and $r_{\text {lim }}=7.0 \AA \AA$. Black-colored contour regions are those for 
$\mu=$ Glu35 of pMLF1 and $r_{\text {lim }}=5.5 \AA$. Green-colored contour regions are those for $\mu=P h e 37$ of pMLF1 and $r_{\text {lim }}=7.0 \AA$. Black arrow with words "O $\gamma$ position of Sep 34 of pMLF1" denotes its position in crystal structure. (B) Closeup around Sep 34 of pMLF1 in the crystal structure. Four contours overlap around the black-line circle. (C) The $\mathrm{O} \gamma$ atomic positions (red spheres show "O $\gamma$ positions of Sep 34 in small-RMSD $D_{\text {lig }}$ confs") of snapshots with $R M S D_{\text {lig }} \leq 1.0 \AA$. The molecular structure shown is the crustal structure.

Figure 10A shows a large region (red contours) for Sep 34 of pMLF1. This region well overlaps high-density spots $E_{1}$ and $E_{2}$ (Figure 6). This result is natural because Sep 34 can form salt bridges when Sep 34 approaches the binding partners.

In contrast, the green contours for Phe 37 of pMLF1 were sparse on the surface of 14-3-3e, which suggests that the hydrophobic interaction of Phe 37 to $14-3-3 \varepsilon$ is weaker than that of Phe 33 (blue contours) to stabilize the complex structure. The black contours for Glu 35 of pMLF1 were localized. Therefore, Glu 35 formed the salt bridge only when Glu 35 reached the binding partner Lys 50 of $14-3-3 \varepsilon$.

Figure 10B portrays a closeup image of Figure 10A, particularly addressing Sep 34 of pMLF1 in the crystal structure. Apparently, the four contours overlapped in a narrow region around the Sep 34 position of the crystal structure, as shown in the figure by a black-line circle with the words "Overlapping region". We emphasize that this narrow region involves the O $\gamma$ atomic position of Sep 34 in the crystal structure. Consequently, the simultaneous formation of the four important intermolecular interactions related to Phe 33, Sep 34, Glu 35, and Phe 37 of pMLF1 are possible only when the $\mathrm{O} \gamma$ atom is near the crystal structure position. As presented in Figure 8, Glu 35 shifted somewhat from the crystal-structure position towards the solvent. It is particularly interesting that the black-contours related to Glu 35 in Figures 10A and 10B are the consequence from the shift of Glu 35. Therefore, it is likely that pMLF conformations with the Glu-35 shift belong to the crystal-structure-like complex.

To check the above conjecture further, we selected snapshots with $R M S D_{\text {lig }} \leq 1.0 \AA$, and displayed the $\mathrm{O} \gamma$-atomic positions of these small-RMSD $D_{\text {lig }}$ snapshots in $3 \mathrm{D}$ real space. In fact, Figure $10 \mathrm{C}$ demonstrates that the $\mathrm{O} \gamma$-atomic positions of those snapshots overlapped well to the crystal-structure position. This result also supports that simultaneous satisfaction of the four intermolecular interactions engenders the crystal-structure-like complex form.

\section{DISCUSSION}

The current study presents a binding process of pMLF1 to 14-3-3e (Figure 11). Before binding to $14-3-3$, pMLF1 exists everywhere around $14-3-3 \varepsilon$ at a low concentration $\left(\rho_{\operatorname{Sep} 34}=0.004 \rho_{0}\right.$; dark green contours). It is presumable from the magenta-colored contours $\left(\rho_{\text {Sep } 34}=0.03 \rho_{0}\right)$ that pMLF1 can contact to four regions of the 14-3-3e surface, $D_{1}, \ldots, D_{4}$, more stably or more frequently than to the other regions. An encounter complex is thereby formed. If pMLF1 binds to $D_{1}$ first, then pMLF1 might form a crystal-structure-like complex quickly by adjustment of the conformation.

Because the sum of the regions $D_{2}+D_{3}+D_{4}$ is greater than $D_{1}$, it is likely that pMLF1 binds more frequently to $D_{2}, D_{3}$, or $D_{4}$ than to $D_{1}$. Free-energy barriers exist among three clusters $D_{1}$, $D_{3}$, and $D_{4}$. Therefore, if pMLF1 contacts to $D_{3}$ or $D_{4}$ first, then the complex should jump over the free-energy barriers to reach $D_{1}$. This motion might take a long time. Otherwise, the encounter complex generated in $D_{3}$ or $D_{4}$ might be dissociated sooner or later.

A scenario for the complex formation presumed from the current study is the following: The residue Phe 33 of pMLF1 might contact the hydrophobic contact partner of $14-3-3 \varepsilon$ even when 
pMLF1 is separate from the binding site of 14-3-3e (Figure 10A). This contact might act as an attractive interaction between PMLF1 and 14-3-3e. Then, if the encounter complex is formed in $D_{2}$ first, then no energy jump is necessary to move to $D_{1}$ because the two clusters are connected smoothly without a free-energy barrier. Remember that pMLF1 has no orientational tendency in $D_{2}$. When pMLF1 moves to $D_{1}$, Sep 34 of pMLF1 meets spot $E_{2}$ and binds to Arg 61 and/or Arg 57 of 14-3-3e. Simultaneously, the molecular orientation of pMLF1 tends to be aligned as in the crystal structure. At that stage, neither the hydrophobic contacts related to Phe 37 of pMLF1 nor the salt bridge related to Glu 35 of pMLF1 might be formed. When pMLF1 goes through the corridor from spot $E_{2}$ to $E_{1}$, the salt-bridge relay takes place: The salt bridge between Sep 34 of pMLF1 and Arg 61 of 14-3-3e is broken. Also, that between Sep 34 of pMLF1 and Arg 130 of 14$3-3 \varepsilon$ is formed. When Sep 34 fluctuates in spot $E_{1}$, which involves the crystal-structure position (white-circle position in Figure 11), Phe 37 forms hydrophobic contacts to 14-3-3e. Simultaneously, Glu 35 forms the salt bridge to Lys 50, although the salt bridge to Lys 123 might not be formed. 


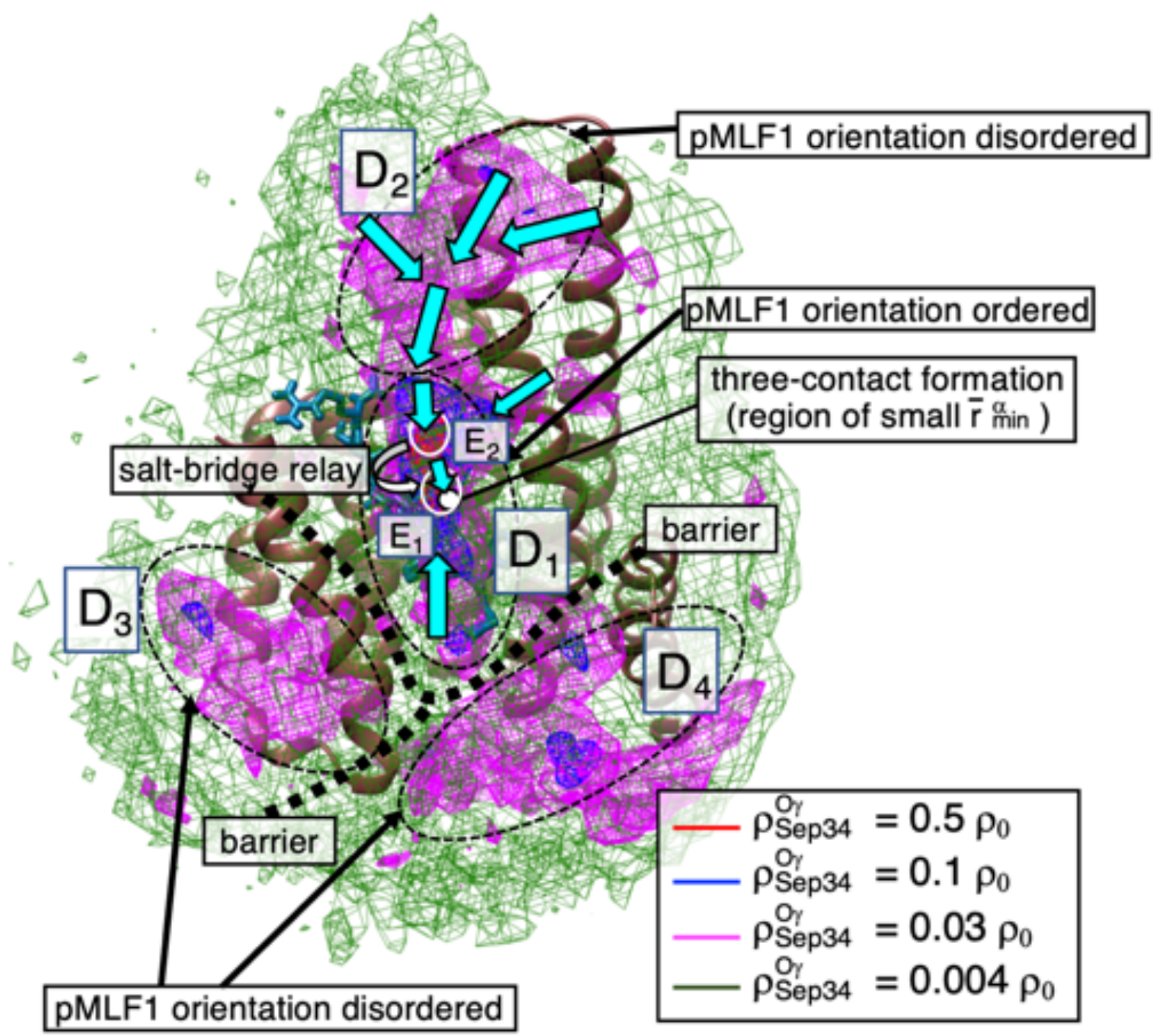

Figure 11. Scheme of binding process of pMLF1 to 14-3-3e. Contour maps represent $\rho_{\text {Sep34 }}(\boldsymbol{r})$ at four density levels as for Figure 5. Clusters $D_{1}, \ldots, D_{4}$ are also from Figure 5. Free-energy barriers are shown by thick broken lines. Cyan-colored arrows represent conformational motions of pMLF1 from cluster $D_{2}$ to $D_{1}$. Molecular orientation of pMLF1 is ordered well in $D_{1}$, although it is less ordered in $D_{2}, D_{3}$, and $D_{4}$. The position where salt-bridge relay takes place is shown by a curved arrow. White open circles are high-density spots $E_{1}$ and $E_{2}$. White filled circles show positions of four intermolecular contacts, quantified by $\bar{r}^{\mu}(\boldsymbol{r})(\mu=$ Phe33, Sep34, Glu35, Phe37), are formed simultaneously.

\section{CONCLUSIONS}

The equilibrated distribution functions derived from the GA-guided $\mathrm{mD}-\mathrm{VcMD}$ suggest a binding process of pMLF1 to 14-3-3e (Figure 11). Furthermore, the distribution provided interesting aspects such as free-energy barriers among $D_{1}, D_{3}$, and $D_{4}$. It also provided pMLF1 orientation ordering and the small ligand-conformational variety in the high-density region and a salt-bridge relay to reach the complex structure similar to the crystal structure. The narrow spot is fundamentally important for crystal structure formation. These aspects are derived naturally from the current simulation without a gap in logic. They are useful to advance other experimental and computational studies. 
In our simulation, Glu 35 shifted somewhat toward solvent. It exhibited large positional fluctuations, even in the high-density spot (Figure 8). These positional shift and large fluctuations were induced by absence of the salt bridge to Lys 123 of 14-3-3e, which is formed in the crystal structure (PDB ID: 3ual). Our simulation study supports experimental data showing that mutation E35A of pMLF1 does not alter $K_{D}$ for the pMLF1/14-3-3e complex.? Results of our simulation also suggest that large fluctuations of Glu 35 affect the hydrophobic interaction of Phe 37 with the sidechain stem of Lys 50. Mutagenesis K50A of 14-3-3e might provide insightful information supporting our inference.

Results of the current study demonstrated that the GA-guided $\mathrm{mD}-\mathrm{VcMD}$ can treat a very flexible ligand molecule without restraining the molecular flexibility. It is noteworthy that this method can search a wide conformational space in which the ligand covers not only the vicinity of the binding site of the receptor, but also regions distant from the binding site. That is true because the RCs were set to search the wide 3D real space. Although the best selection of the reaction coordinates is not known a priori, the overlap of basins in the RC space is, in general, resolved by increasing the space dimensionality. For this reason, the introduction of multiple RCs was useful in this study to sample the highly flexible chain, pMLF1, binding to the receptor. In contrast, incrementation of the space dimensionality makes sampling data sparse in multi-dimensional RC space. This study has demonstrated that $3 \mathrm{D}$ is appropriate as a tradeoff between the basin-overlap resolution and sparse sampling.

The molecular binding scheme for an intrinsically disordered region/protein (IDR/IDP) has variety: Coupled folding and binding ${ }^{56,58}$ fuzzy complexes, ${ }^{59}$ multimodal complexes, ${ }^{53}$ and dynamic complexes. ${ }^{60}$ The current study demonstrated that pMLF1 exists in two high-density spots $E_{1}$ and $E_{2}$ (Figure 6A). These multiple-state features might also be related to the property of IDR/IDP.

Recently, to investigate intermolecular interactions, we performed multicanonical molecular dynamics (McMD) of an intrinsically disordered peptide, p53 C-terminal domain (p53CTD), binding to its receptor protein (S100B) ${ }^{37}$ It is particularly interesting that results of this study suggest that the degree of disorder of p53CTD increases when binding to S100B. We designated this emerging state upon binding an extra-disorder state. For this study, we analyzed whether the extra-disorder state existed or not by comparing the ligand conformational variety between the bound and unbound states. Nevertheless, we were unable to clarify any clue to the extra-disordered state because the upper limits of $40 \AA$ for RCs (Table S2) were still too short to sample pMLF1 conformations entirely free from 14-3-3e: that is, a pMLF1 snapshot that was not touching 14-3$3 \varepsilon$ can achieve touching by rotating pMLF1 (data not shown). Therefore, sampling of the unfolded state was insufficient. As a subject of future study, we set the RC limits to values that are sufficiently large to generate a ligand completely free from the receptor. Furthermore, it is noteworthy that this increment of RCs leads the GA-guided $\mathrm{mD}-\mathrm{VcMD}$ to calculate $K_{D}$ directly by calculating the probabilities for both the bound and free states.

The density contour map obtained from the current study can be expected to produce many insights. For instance, a molecular modification that changes the corridor width between spot $E_{1}$ and $E_{2}$ alters the kinetic constants. It therefore affects the complex association and dissociation constants. The current simulation also suggests a reason why the mutation E35A did not alter $K_{D}$. These insights might pave the way to new approaches for drug design. We emphasize that the large-scale conformational ensemble equilibrated at a room temperature, which covers the free, encounter-complex, and the most-stable states, is useful to widen the research area for drug discovery. 


\section{ACKNOWLEDGEMENTS}

The authors thank Prof. Norio Ozaki in Nagoya University Graduate School of Medicine for his valuable discussion of the functional roles of the 14-3-3 proteins which interact with the phosphorylated sequences. J. H. was supported by JSPS KAKENHI Grant No. 16K05517 and by the Development of Core Technologies for Innovative Drug Development based upon IT from Japan Agency for Medical Research and Development, AMED. J. H. and K. K. were supported by the HPCI System Research Project (Project IDs: hp190017, hp190018, hp190027, hp200025,

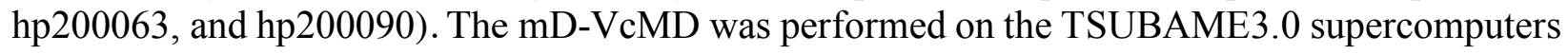
at the Tokyo Institute of Technology. K. K. was also supported by JSPS KAKENHI Grant No. 16K18526. H. N. was supported by a Grant-in-Aid for Scientific Research on Innovative Areas (24118008) and a Grant-in-Aid for Challenging Exploratory Research (16K14711) from JSPS. N. K. was supported by JSPS KAKENHI Grant (Number JP20H03229). It was performed in part under the Cooperative Research Program of the Institute for Protein Research, Osaka University, CR-19-05 and CR-20-05.

\section{REFERENCES}

(1) Yaffe, M. B.; Rittinger, K.; Volinia, S.; Caron, P. R.; Aitken, A.; Leffers, H.; Gamblin, S. J.; Smerdon, S. J.; Cantley, L. C. The Structural Basis for 14-3-3: Phosphopeptide Binding Specificity. Cell 1997, 91, 961-971.

(2) Yaffe, M. B. How Do 14-3-3 Proteins Work? -- Gatekeeper Phosphorylation and the Molecular Anvil Hypothesis. FEBS Lett. 2002, 513, 53-57.

(3) Johnson, C.; Crowther, S.; Stafford, M. J.; Campbell, D. G.; Toth, R.; MacKintosh, C. Bioinformatic and Experimental Survey of 14-3-3-Binding Sites. Biochem. J. 2010, 427, 6978.

(4) Morrison D. K. The 14-3-3 Proteins: Integrations of Diverse Signaling Cues That Impact Cell Fate and Cancer Development. Trends. Cell Biol. 2009, 19, 16-23.

(5) Stevers, L. M.; Sijbesma, E.; Botta, M.; MacKintosh, C.; Obsil, T.; Landrieu, I.; Cau, Y.; Wilson, A. J.; Karawajczyk, A.; Eickhoff, J.; Davis, J.; Hann, M.; O’Mahony, G.; Doveston, R. G.; Brunsveld, L.; Ottmann, C. Modulators of 14-3-3 Protein-Protein Interactions, J. Med. Chem. 2018, 61, 3755-3778.

(6) Kawabata, T.; Nishikawa. K. Protein Structure Comparison Using the Markov Transition Model of Evolution. Proteins 2000, 41, 108-122.

(7) Molzan, M.; Weyand, M.; Rose, R.; Ottmann, C. Structural Insights of the MLF1/14-3-3 Interaction. FEBS J. 2012, 279, 563-571.

(8) Kimura, H.; Tsuboi, D.; Wang, C.; Kushima, I.; Koide, T.; Ikeda, M.; Iwayama, Y.; Toyota, T.; Yamamoto, N.; Kunimoto, S.; Nakamura, Y.; Yoshimi, A.; Banno, M.; Xing, J.; Takasaki, Y.; Yoshida, M.; Aleksic, B.; Uno, Y.; Okada, T.; Iidaka, T.; Inada, T.; Suzuki, M.; Ujike, H.; Kunugi, H.; Kato, T.; Yoshikawa, T.; Iwata, N.; Kaibuchi, K.; Ozaki. N. Identification of Rare, 
Single-Nucleotide Mutations in NDE1 and Their Contributions to Schizophrenia Susceptibility. Schizophr Bull. 2015, 41, 744-753.

(9) Matsumoto, N.; Yoneda-Kato, N.; Iguchi, T.; Kishimoto, Y.; Kyo, T.; Sawada, H.; Tatsumi, E.; Fukuhara, S. Elevated MLF1 Expression Correlates with Malignant Progression from Myelodysplastic Syndrome. Leukemia 2000, 14, 1757-1765.

(10) Lim, R.; Winteringham, L.N.; Williams, J.H.; McCulloch,R.K.; Ingley, E.; Tiao, J.Y-H.; Lalonde, J-P.; Tsai, S.; Tilbrook, P. A.; Sun, Y.; Wu, X.; Morris, S.W.; Klinken, S. P. MADM, a novel adaptor protein that mediates phosphorylation of the 14-3-3 binding site of myeloid leukemia factor 1. J Biol Chem. 2002, 277, 40997-41008.

(11) Winteringham, L. N.; Endersby, R.; Kobelke, S.; McCulloch, R. K.; Williams, J. H.; Stillitano, J.; Cornwall, S. M.; Ingley, E.; Klinken, S.P. Myeloid Leukemia Factor 1 Associates with a Novel Heterogeneous Nuclear Ribonucleoprotein U-Like Molecule. J. Biol. Chem. 2006, 281, 38791-38800.

(12) Molzan M.; Ottman C. Subcellular Localization of Full-length Human Myeloid Leukemia Factor 1 (MLF1) Is Independent of 14-3-3 proteins. Cellular \& Molecular Biology Letters 2013, 18, 137-148.

(13) Berg, B. A.; Neuhaus, T. Multicanonical Ensemble: A New Approach to Simulate First-Order Phase Transitions. Phys. Rev. Lett. 1992, 68, 9-12.

(14) Hansmann, U. H. E.; Okamoto, Y. Prediction of Peptide Conformation by Multicanonical Algorithm: New Approach to the Multiple-Minima Problem. J. Comput. Chem. 1993, 14, 1333-1338.

(15) Kidera, A. Enhanced Conformational Sampling in Monte Carlo Simulations of Proteins: Application to a Constrained Peptide. Proc. Natl. Acad. Sci. USA 1995, 92, 9886-9889.

(16) Nakajima, N.; Nakamura, H.; Kidera, A. Multicanonical Ensemble Generated by Molecular Dynamics Simulation for Enhanced Conformational Sampling of Peptides. J. Phys. Chem. B 1997, 101, 817-824.

(17) Hukushima, K.; Nemoto, K.. Exchange Monte Carlo Method and Application to Spin Glass Simulations. J. Phys. Soc. Jpn. 1996, 65, 1604-1608.

(18) Sugita, Y.; Okamoto, Y. Replica-Exchange Molecular Dynamics Method for Protein Folding. Chem. Phys. Lett. 1999, 314, 141-151.

(19) Lee, J. New Monte Carlo Algorithm: Entropic Sampling. Phys. Rev. Lett. 1993, 71, 211-214.

(20) Wang, F.; Landau, D. P. Efficient, Multiple-Range Random Walk Algorithm to Calculate the Density of States. Phys. Rev. Lett. 2001, 86, 2050-2053.

(21) Darve, E.; Pohorille, A. Calculating Free Energies Using Average Force. J. Chem. Phys. 2001, 115, 9169-9183. 
(22) Laio, A.; Parrinello, M. Escaping Free-Energy Minima. Proc. Natl. Acad. Sci. USA 2002, 99, $12562-12566$.

(23) Iba, Y.; Chikenji, G.; Kikuchi, M. Simulation of Lattice Polymers with Multi-Self-Overlap Ensemble. Phys. Soc. Jpn. 1998, 67, 3327-3330.

(24) Swendsen, R. H.; Wang, J. S. Replica Monte Carlo Simulation of Spin Glasses. Phys. Rev. Lett. 1986, 57, 2607-2609.

(25) Fukunishi, H.; Watanabe, O.; Takada, S. On the Hamiltonian Replica Exchange Method for Efficient Sampling of Biomolecular Systems: Application to Protein Structure Prediction. $J$. Chem. Phys. 2002, 116, 9058-9067.

(26) Higo, J.; Dasgupta, B., Mashimo T.; Kasahara, K.; Fukunishi, Y., Nakamura, H. VirtualSystem-Coupled Adaptive Umbrella Sampling to Compute Free-Energy Landscape for Flexible Molecular Docking. J. Comput. Chem. 2015, 36, 1489-1501.

(27) Higo, J.; Kasahara, K.; Dasgupta, B.; Nakamura, H. Enhancement of Canonical Sampling by Virtual-State Transitions. J. Chem. Phys. 2017, 146, 044104.

(28) Paine, G. H.; Scheraga, H. A. Prediction of the Native Conformation of a Polypeptide by a Statistical-Mechanical Procedure. I. Backbone Structure of Enkephalin. Biopolymers 1987, 26, 1391-1436.

(29) Mezei, M. Adaptive Umbrella Sampling: Self-Consistent Determination of the NonBoltzmann Bias. J. Comput. Phys. 1987, 68, 237-248.

(30) Higo, J.; Nakamura, H. Virtual States Introduced for Overcoming Entropic Barriers in Conformational Space. Biophysics 2012, 8, 139-144.

(31) Higo, J.; Umezawa, K.; Nakamura, H. A Virtual-System Coupled Multicanonical Molecular Dynamics Simulation: Principle and Its Application to Free-Energy Landscape of ProteinProtein Interaction with an All-Atom Model in Explicit Solvent. J. Chem. Phys. 2013, 138, 184106.

(32) Dasgupta, B.; Higo J.; Nakamura, H. Faster Binding Free-Energy Landscape Calculation by Virtual-State Coupled Adaptive Umbrella Sampling. Biophys. J. 2016, 110, 55a.

(33) Terakawa, T.; Higo, J.; Takada, S. Multi-Scale Ensemble Modeling of Modular Proteins with Intrinsically Disordered Linker Regions: Application to p53. Biophys. J. 2014, 107, 721-729.

(34) Okuda, M.; Higo, J.; Komatsu, T.; Konuma, T.; Sugase, K., Nishimura, Y. Dynamics of the Extended String-Like Interaction of TFIIE with the p62 Subunit of TFIIH. Biophys. J. 2016, $111,950-962$.

(35) Dasgupta, B.; Nakamura, H.; Higo J. Flexible Binding Simulation by a Novel and Improved Version of Virtual-System Coupled Adaptive Umbrella Sampling. Chem. Phys. Lett. 2016, $662,327-332$. 
(36) Iida, S.; Mashimo, T.; Kurosawa, T.; Hojo, H.; Muta, H.; Goto, Y.; Fukunishi, Y.; Nakamura, H.; Higo, J. Variation of Free-Energy Landscape of the p53 C-terminal Domain Induced by Acetylation: Enhanced Conformational Sampling. J. Comput. Chem. 2016, 37, 2687-2700.

(37) Iida, S.; Kawabata T.; Kasahara, K.; Nakamura, H.; Higo, J. Multimodal Structural Distribution of the p53 C-Terminal Domain Upon Binding to S100B via a Generalised Ensemble Method: From Disorder to Extra-Disorder. J. Chem. Theory Comput. 2019, 15, 2597-2607.

(38) Hayami, T.; Higo, J.; Nakamura, H.; Kasahara, K. Multidimensional Virtual-System Coupled Canonical Molecular Dynamics to Compute Free-Energy Landscapes of Peptide Multimer Assembly. J. Comput. Chem. 2019, 40, 2453-2463.

(39) Higo, J.; Kasahara, K.; Wada, M.; Dasgupta, B.; Kamiya, N.; Hayami, T.; Fukuda, I.; Fukunishi, Y.; Nakamura, H. Free-Energy Landscape of Molecular Interactions between Endothelin 1 and Human Endothelin Type B Receptor: Fly-Casting Mechanism. Protein Eng. Des. Sel. 2019, 32, 297-308.

(40) Higo, J.; Kusaka, A.; Kasahara, K.; Kamiya, N.; Fukuda, I.; Mori, K.; Hata, Y.; Fukunishi, F. arXiv: 2006.06950, 2020.

(41) Higo, J.; Kamiya, N.; Sugihara, T.; Yonezawa, Y.; Nakamura, H. Verifying Trivial Parallelization of Multicanonical Molecular Dynamics for Conformational Sampling of a Polypeptide in Explicit Water. Chem. Phys. Lett. 2009, 473, 326-329.

(42) Ikebe, J.; Umezawa, K.; Kamiya, N.; Sugihara, T.; Yonezawa, T., Takano, Y.; Nakamura, H.; Higo, J. Theory for Trivial Trajectory Parallelization of Multicanonical Molecular Dynamics and Application to a Polypeptide in Water. J. Comput. Chem. 2011, 32, 1286-1297.

(43) Kasahara, K.; Ma, B.; Goto, K.; Dasgupta, B.; Higo, J.; Fukuda, I.; Mashimo, T.; Akiyama, Y.; Nakamura, H. myPresto/Omegagene: a GPU-Accelerated Molecular Dynamics Simulator Tailored for Enhanced Conformational Sampling Methods with a Non-Ewald Electrostatic Scheme. Biophys. Physicobiol. 2016, 13, 209-216.

(44) Ryckaert, J.-P.; Ciccotti, G.; Berendsen, H. J. C. Numerical Integration of the Cartesian Equations of Motion of a System with Constraints: Molecular Dynamics of $n$-Alkanes. $J$. Comput. Phys. 1977, 23, 327-341.

(45) Berendsen, H. J. C.; Postma, J. P. M.; van Gunsteren, W. F.; DiNola, A.; Haak, J. R. Molecular Dynamics with Coupling to an External Bath. J. Chem. Phys. 1984, 81, 3684-3690.

(46) Kamiya, N.; Fukuda, I.; Nakamura, H. Application of Zero-Dipole Summation Method to Molecular Dynamics Simulations of a Membrane Protein System. Chem. Phys. Lett. 2013, $568-569,26-32$.

(47) Fukuda, I.; Kamiya, N.; Yonezawa, Y.; Nakamura, H. Simple and Accurate Scheme to Compute Electrostatic Interaction: Zero-Dipole Summation Technique for Molecular System and Application to Bulk Water. J. Chem. Phys. 2012, 137, 054314. 
(48) Fukuda, I.; Yonezawa, Y.; Nakamura, H. Molecular Dynamics Scheme for Precise Estimation of Electrostatic Interaction via Zero-Dipole Summation Principle. J. Chem. Phys.2011, 134, 164107.

(49) Morishita, T. Fluctuation Formulas in Molecular-Dynamics Simulations with the Weak Coupling Heat Bath. J. Chem. Phys. 2000, 113, 2976-2982.

(50) Kamiya, N.; Watanabe, Y. S.; Ono, S.; Higo, J. AMBER-based hybrid force field for conformational sampling of polypeptides. Chem. Phys. Lett. 2005, 401, 312-317.

(51) Jorgensen, W. L.; Chandrasekhar, J.; Madura, J. D.; Impey, R. W.; Klein, M. L. Comparison of Simple Potential Functions for Simulating Liquid Water. J. Chem. Phys. 1983, 79, 926935.

(52) Joung, I. S.; Cheatham, T. E. Determination of Alkali and Halide Monovalent Ion Parameters for Use in Explicitly Solvated Biomolecular Simulations. J. Phys. Chem. B 2008, 112, 90209041.

(53) Kasahara, K.; Shiina, M.; Higo, J.; Ogata, K.; Nakamura, H. Phosphorylation of an Intrinsically Disordered Region of Ets1 Shifts a Multi-modal Interaction Ensemble to an Autoinhibitory State. Nucleic Acids Research 2018, 46, 2243-2251.

(54) Arai, M. Unified Understanding of Folding and Binding Mechanisms of Globular and Intrinsically Disordered Proteins. Biophys. Rev. 2018, 10, 163-181.

(55) Shoemaker, B. A.; Portman, J. J.; Wolynes, P. G. Speeding Molecular Recognition by Using the Folding Funnel: The Fly-Casting Mechanism. Proc. Natl. Acad. Sci. USA 2000, 97, 88688873.

(56) Sugase, K.; Dyson, H. J.; Wright, P. E. Mechanism of Coupled Folding and Binding of an Intrinsically Disordered Protein. Nature 2007, 447, 1021-1025.

(57) Kofuku, Y.; Yoshiura, C.; Ueda, T.; Terasawa, H.; Hirai, T.; Tominaga, S.; Hirose, M.; Maeda, Y.; Takahashi, H.; Terashima, Y.; Matsushima, K.; Shimada, I. Structural Basis of the Interaction between Chemokine Stromal Cell-derived Factor-1/CXCL12 and Its G-proteincoupled Receptor CXCR4. J. Biol. Chem. 2009, 284, 35240-35250.

(58) Wright, P. E.; Dyson, H. J. Intrinsically Unstructured Proteins: Re-assessing the Protein Structure-function Paradigm. J. Mol. Biol. 1999, 293, 321-331.

(59) Tompa, P.; Fuxreiter, M. Fuzzy Complexes: Polymorphism and Structural Disorder in Protein-Protein Interactions. Trends Biochem. Sci. 2008, 33, 2-8.

(60) Milles, S.; Salvi, N.; Blackledge, M.; Jensen, M. R. Characterization of Intrinsically Disordered Proteins and Their Dynamic Complexes: From in Vitro to Cell-like Environments. Progress in Nuclear Magnetic Resonance Spectroscopy 2018, 109, 79-100. 
Supporting Information

\section{Molecular interaction mechanism of a 14-3-3 protein with a phosphorylated peptide elucidated by enhanced conformational sampling}

Junichi Higo, ${ }^{1, *}$ Takeshi Kawabata, ${ }^{2}$ Ayumi Kusaka, ${ }^{1}$ Kota Kasahara, ${ }^{3}$ Narutoshi Kamiya, ${ }^{1}$ Ikuo Fukuda, ${ }^{1}$ Kentaro Mori, ${ }^{1,4}$ Yutaka Hata, ${ }^{1}$ Yoshifumi Fukunishi, ${ }^{5}$ Haruki Nakamura. ${ }^{2}$

${ }^{1}$ Graduate School of Simulation Studies, University of Hyogo, 7-1-28 Minatojima Minami- machi, Chuo-ku, Kobe, Hyogo 650-0047, Japan

${ }^{2}$ Institute for Protein Research, Osaka University, 3-2 Yamada-oka, Suita, Osaka 565-0871, Japan

${ }^{3}$ College of Life Sciences, Ritsumeikan University, 1-1-1 Noji-higashi, Kusatsu, Shiga 525-8577, Japan

${ }^{4}$ Maizuru College, 234 Shiroya, Maizuru, Kyoto, 625-8511 Japan

${ }^{5}$ Cellular and Molecular Biotechnology Research Institute, National Institute of Advanced Industrial Science and Technology (AIST), 2-3-26, Aomi, Koto-ku, Tokyo, 135-0064, Japan.

*Electronic mail: higo@protein.osaka-u.ac.jp 


\section{Figure S1}

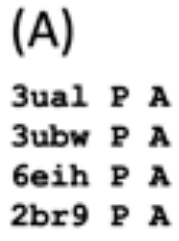

(B)
RSFPSEPFG RSFPSEPF SIPSR RQRPSAP
(C)

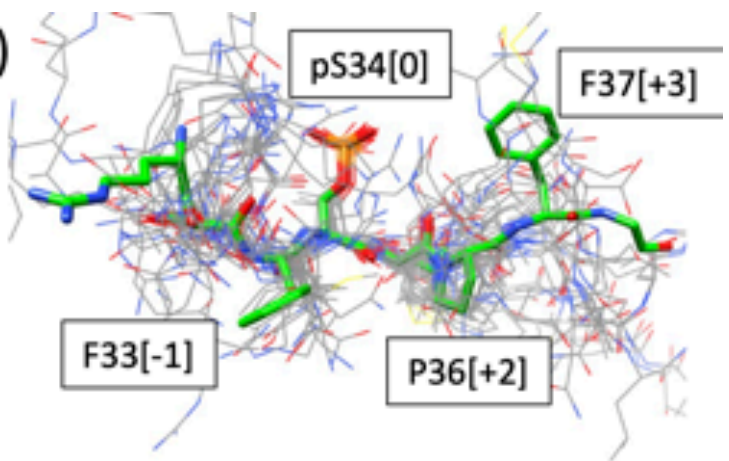

(D)

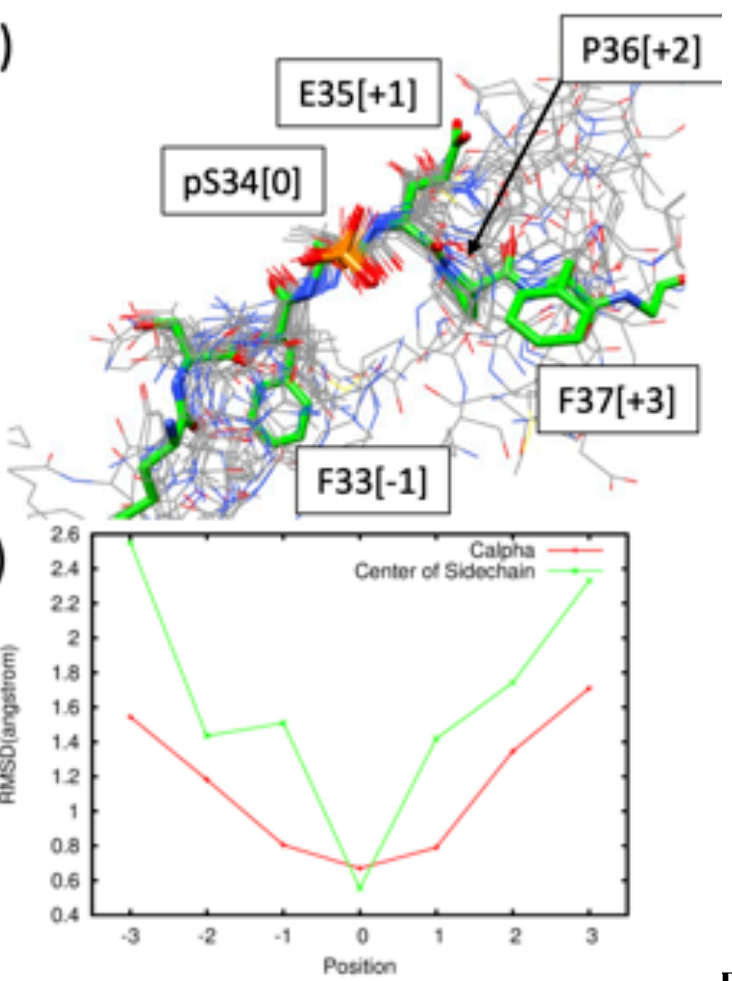

Figure S1. (A) Four structurally known phosphorylated peptides bound to human 14-3-3e protein. PDB IDs, chain IDs of binding peptide, chain IDs of 14-3-3 protein and amino acid sequences of binding peptides are shown. pS and pT are phosphorylated serine and threonine residues, respectively. (B) 25 structurally known phosphorylated peptides bound to human $14-3-3 \zeta$ protein. Sequence identity between $14-3-3 \varepsilon$ and $14-3-3 \zeta$ is $67 \%$. (C) A view of the superimposed $3 \mathrm{D}$ structures of binding peptides to $14-3-3 \varepsilon$ or $14-3-3 \zeta$ proteins. They were superimposed by their bound 3D structures of 14-3-3 proteins on A chain (14-3-3e) of PDB ID:3ual, using the program MATRAS. ${ }^{[1]}$ The peptide structure of $\mathrm{P}$ chain (i.e., pMLF1) of PDB ID:3ual is shown in green. The residue numbering accords to UniProt (M.MLF1_HUMAN; https://www.uniprot.org/uniprot/P58340). Numbers in square brackets are residue ordinal numbers counted from the phosphorylated residue. (D) A view of the superimposed 3D structures from a different orientation. (E) RMSD plots of the superimposed 28 peptides with the P chain of PDB ID:3ual. Red line shows RMSDs of the corresponding $\mathrm{C} \alpha$ atoms, and the green line those of center of sidechain atoms.

\section{Figure S2}




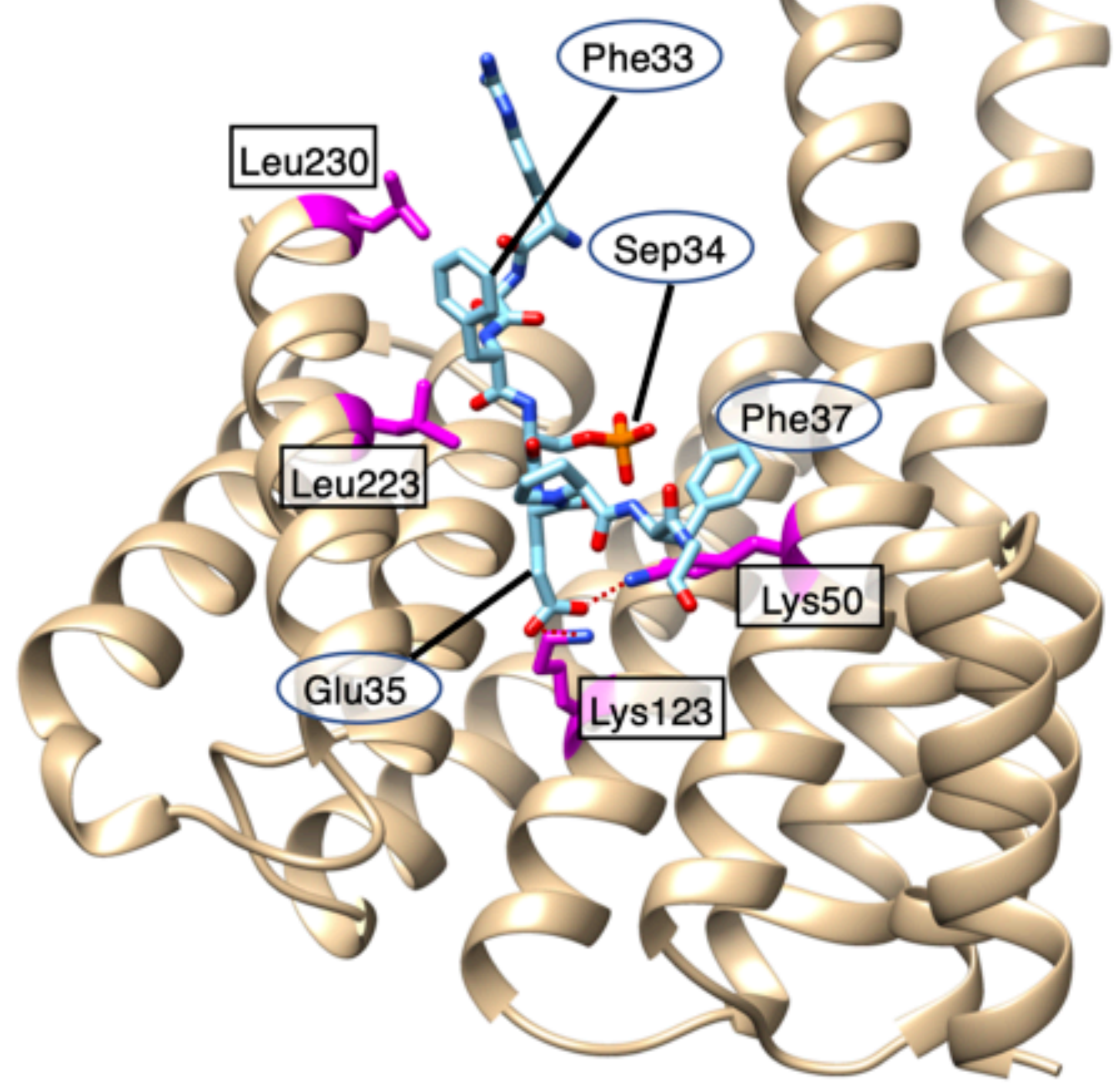

Figure S2. 14-3-3e/MLF1 complex structure solved by X-ray crystallography, where MLF1 and 14-3-3e are shown in cyan and ocher, respectively. Residues Phe 33, Sep 34, Glu 35, and Phe 37 belong to MLF1, and Lys 50, Lys 123, Leu 223, and Leu 230, shown by magenta, do to 14-3-3e. Salt bridges from Glu 35 of MLF1 to Lys 50 and Lys 123

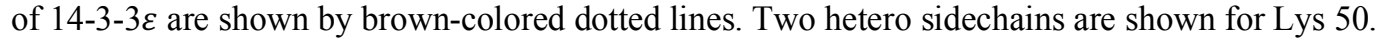

\section{Section 1. Basic method to define a reaction coordinate $(\mathrm{RC})$}

Consider two atom groups $G_{h}^{A}$ and $G_{h}^{B}(h=\alpha, \beta, \gamma)$ in a molecular system. The $\mathrm{RC}, \lambda^{(h)}$, is defined by the distance between centers of mass of $G_{h}^{A}$ and $G_{h}^{B}$ (Fig. S3). Superscripts $A$ and $B$ indicate simply that two atom groups are pairing to define $\lambda^{(h)}$, and then, one can exchange the superscripts as: $G_{h}^{A} \rightarrow G_{h}^{B}$ and $G_{h}^{B} \rightarrow G_{h}^{A}$ without changing the value of $\lambda^{(h)}$. 


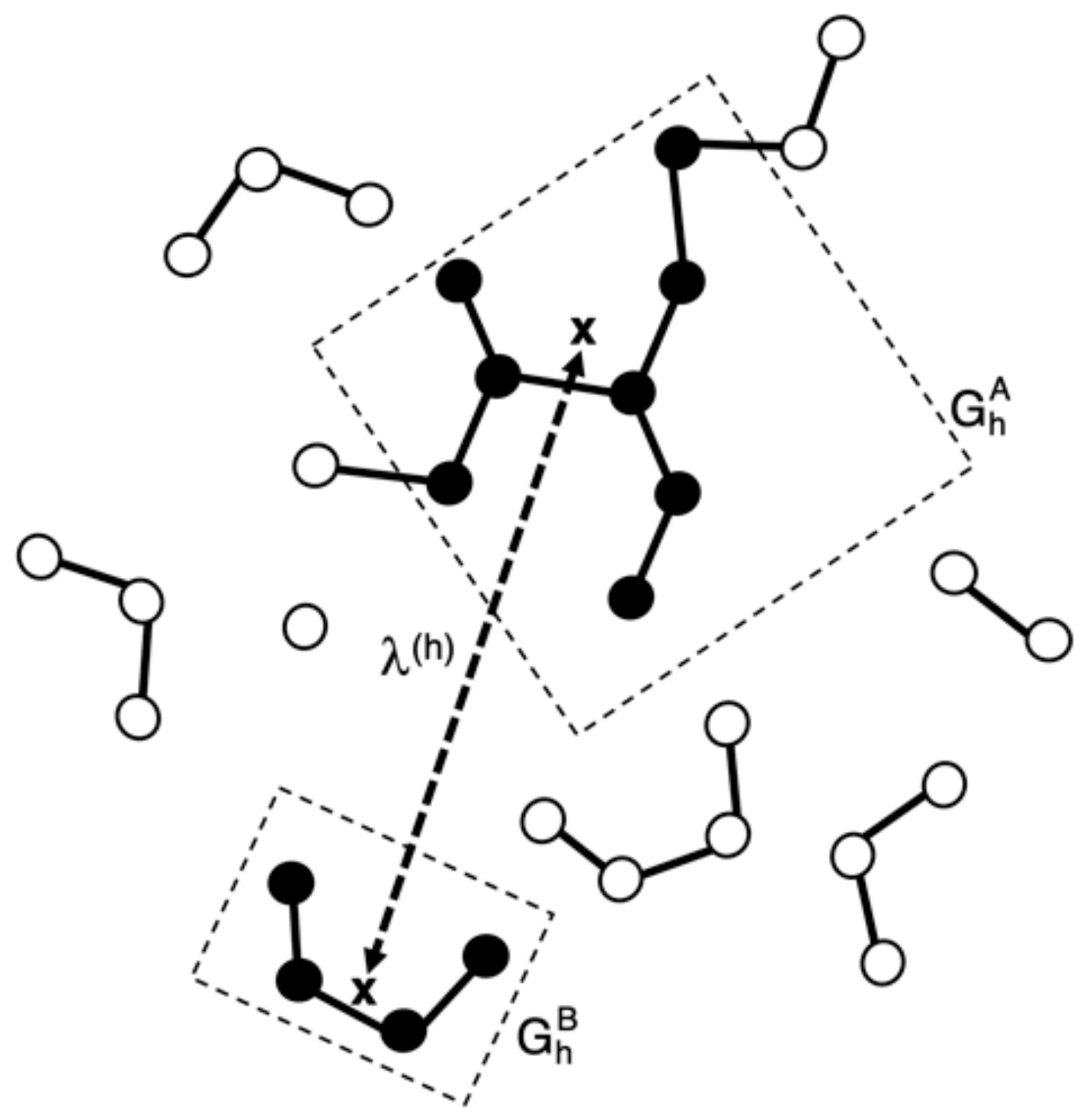

Figure S3. Two atom groups, $G_{h}^{A}$ and $G_{h}^{B}$, are indicated by two rectangles, where in-group atoms are shown by black filled circles. Center of mass of each atom group is presented by a cross. The distance between the two centers of mass is $\lambda^{(h)}$ (broken-line with arrows). 


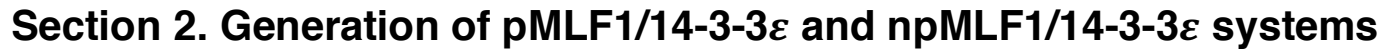

To generate the pMLF1/14-3-3e system, first, the X-ray crystal structure of the complex of 14-3$3 \varepsilon$ with pMLF1 (i.e., MLF1 phosphorylated at the 34-th serine residue; see the main text for the amino-acid sequence), was taken from the PDB data (ID: 3 ual). ${ }^{2}$ Residue ordinal numbers used in this study accord to those in the paper, ${ }^{2}$ which are not the same as those used in the PDB data. The receptor 14-3-3e forms a homodimer in the unbinding state, as shown in Fig. S4A schematically. The ligand pMLF1 binds to each of the two 14-3-3e molecules (Fig. S4B), where Sep 34 (the 34th serine residue phosphorylated) of pMLF1 forms salt bridges to Arg 57 and Arg 130 of 14-3-3e in the crystal structure (Fig. S2). To reduce the computational load, in the present study, we used

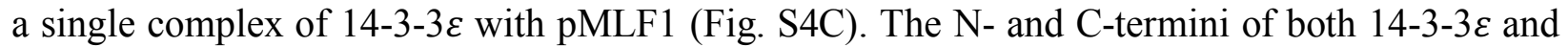
pMLF1 were capped by acetyl and N-methyl groups, respectively. The single complex taken from 3ual was immersed in a periodic water box $\left(86.2 \times 86.2 \times 90.8 \AA^{3}\right)$ under periodic boundary condition, and sodium and chlorine ions were introduced to neutralize the net charge of the entire system at a physiological ionic concentration. The resultant system consists of 65,776 atoms $(3,700$ atoms for 14-3-3e; 137 atoms for pMLF1; 20,612 molecules for water; 46 chlorine ions and 57 for sodium ions).

(A)

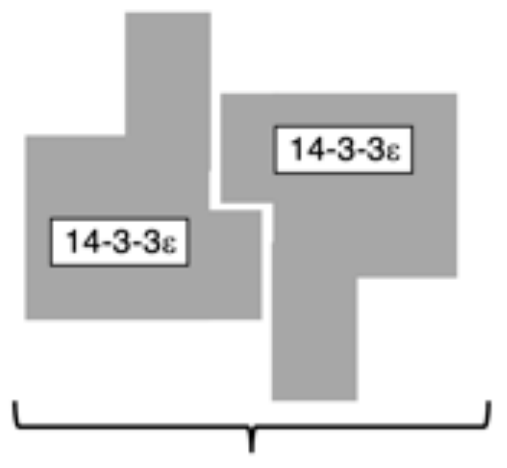

Dimer of $14-3-3 \varepsilon$
(B)

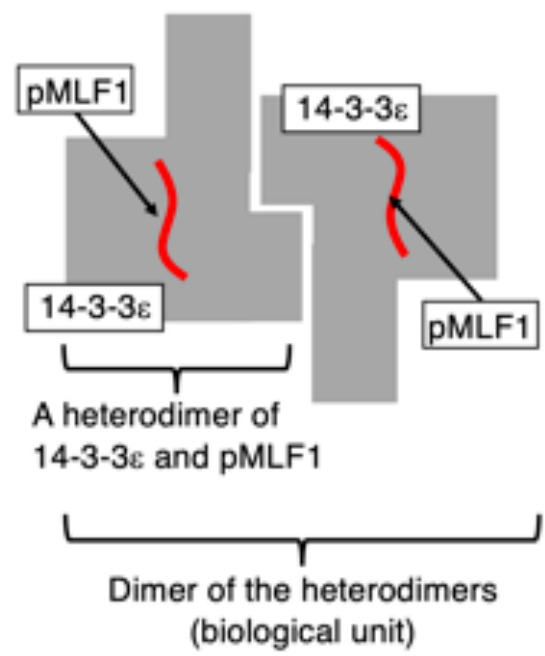

(C)

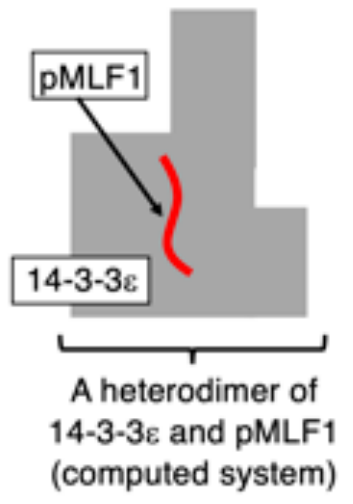

Figure S4.

Shaded polygon and red-colored string are receptor $14-3-3 \varepsilon$ and ligand pMLF1, respectively. (A) 14-3-3 $\varepsilon$ dimer in

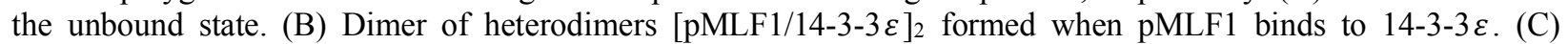
Computed system.

Although pMLF1 used for the experiment ${ }^{2}$ was a segment of 14 residues long, the two $\mathrm{N}$ - and four $\mathrm{C}$-terminal residues were not determined in the experiment. Then, we treated the eight-residue determined segment in the present study. The receptor 14-3-3 $\varepsilon$ consists of 230 amino-acid residues (see PDB: 3 ual). We refer to the current system consisting of the capped pMLF1 and 14-3-3 $\varepsilon$ as the pMLF1/14-3-3 $\varepsilon$ system, whether the two molecules form a complex or not during simulation.

We also computed a system of 14-3-3 $\varepsilon$ and npMLF1 (the non-phosphorylated MLF1; see the main text for the amino-acid sequence), and we refer to this system as an npMLF1/14-3-3 $\varepsilon$ system. This system was generated by replacing Sep 34 of pMLF1 in the pMLF1/14-3-3 $\varepsilon$ system by serine. Then, the numbers of ions and water molecules were modified to neutralize the net charge of the system. The resultant system consists of 65,771 atoms $(3,700$ atoms for $14-3-3 \varepsilon ; 134$ atoms for npMLF1; 20,612 molecules for water; 46 chlorine ions and 55 for sodium ions). 
The pMLF1/14-3-3e complex structure generated above is the same as the crystal structure. On the other hand, a stable complex is not reported for the npMLF1/14-3-3e system. However, the npMLF1/14-3-3 $\varepsilon$ complex structure generated above adopts the crystal structure of the

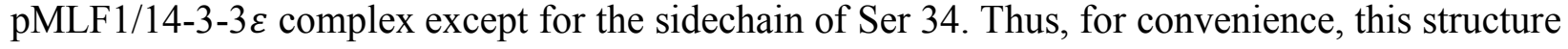
is also called the crystal structure in this study.

For each of the pMLF1/14-3-3 $\varepsilon$ and npMLF1/14-3-3 $\varepsilon$ systems, after a short energy minimization of the complex structure, a short constant-volume and constant-temperature (300 K) simulation (NVT simulation) was performed. Then, the system size was relaxed by a constantpressure (1 atm) and constant-temperature simulation (NPT simulation). The resultant box size was $84.70 \times 84.67 \times 89.24 \AA^{3}$ and $84.72 \times 84.69 \times 89.27 \AA^{3}$ for the pMLF1/14-3-3 $\varepsilon$ and npMLF1/14-3-3 $\varepsilon$ systems, respectively.

We aim to search the conformational space of both systems starting from randomized ligand's conformations of pMLF1 or npMLF1. However, the obtained structures from the NPT simulation were still close to the crystal structure (data not shown). Thus, we generated the randomized conformations as explained in section 3 of SI below. The word "initial conformation" used in the present study represents the randomized conformation where pMLF1 or npMLF1 is apart from the pMLF1 binding site of $14-3-3 \varepsilon$.

\section{Section 3. Generation of initial conformations}

As shown in Fig. 1 of main text and Table S1, we introduced three RCs, $\lambda^{(\alpha)}, \lambda^{(\beta)}$, and $\lambda^{(\gamma)}$. To generate randomized conformations of ligand (pMLF1 or npMLF1), we performed a simulation with applying weak repulsive forces along the three RCs so that the RCs fell in the ranges of $38 \AA \leq \lambda^{(h)} \leq 40 \AA(h=\alpha, \beta, \gamma)$. Then, for both systems, we performed 256 runs starting from the conformation obtained from the NPT simulation with different initial velocities of atoms, and used the final snapshots for the initial conformations of the first iteration of GA-guided mD-VcMD. In the resultant snapshots, pMLF1 or npMLF1 was distant from the binding site of 14-3-3e, which were slightly contacting to or unbound from 14-3-3 $\varepsilon$. Figure S5 of SI displays some of the initial conformations randomly picked from the 256 initial conformations. 


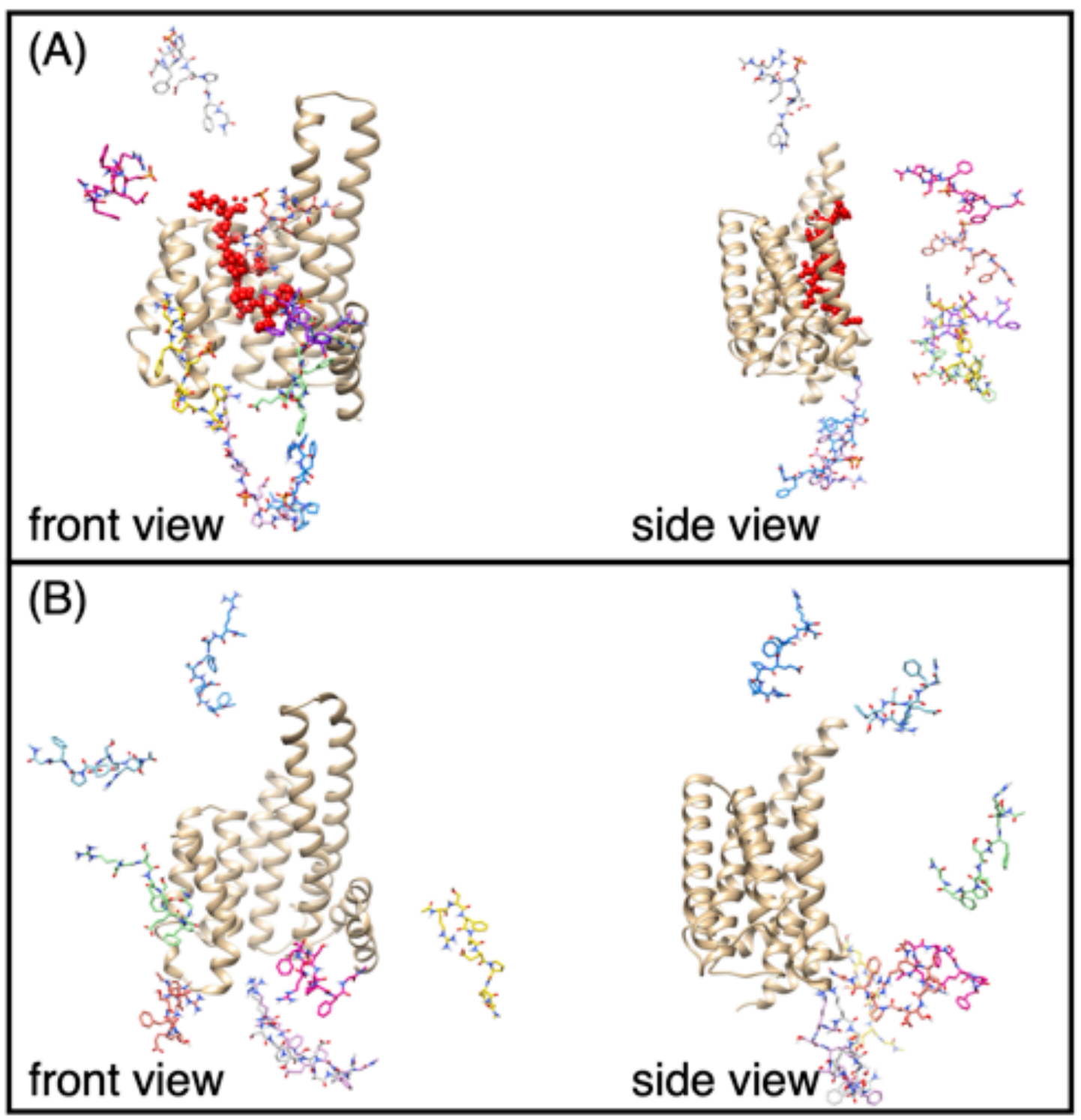

Figure S5. The initial conformations of GA-guided mD-VcMD for (A) pMLF1/14-3-3e and (B) npMLF1/14-3-3 $\varepsilon$ systems. Eight conformations picked randomly from 256 initial ones are shown. Red-colored model in panel (A) is pMLF1 in the crystal structure. Note that this red-colored model is not used for the initial conformation of the GAguided $\mathrm{mD}-\mathrm{VcMD}$. Solvent was omitted in this figure.

\section{Section 4. Iterative $\mathrm{mD}-\mathrm{VcMD}$ simulations and parameters used}

GA-guided $\mathrm{mD}-\mathrm{VcMD}$ consists of iterative simulations, through which the conformational ensemble converges on an equilibrated one at a simulation temperature $(300 \mathrm{~K})$. We repeated 30 and 14 iterations for the pMLF1/14-3-3 $\varepsilon$ and npMLF1/14-3-3 $\varepsilon$ systems, respectively. To sample carefully the electrostatic interactions between Sep 34 of pMLF1 and charged residues in 14-3-3e, we did more iterations for pMLF1/14-3-3 $\varepsilon$ than npMLF1/14-3-3 $\varepsilon$. For both systems, a set of 256 runs were bundled in an iteration. Each of 256 runs in an iteration was performed for $2 \times 10^{6}$ steps $(4 \mathrm{~ns})$. Thus, the total simulation length was $30.720 \mu \mathrm{s}(=30 \times 256 \times 4 \mathrm{~ns})$ and $14.336 \mu \mathrm{s}(=14$ 
$\times 256 \times 4 \mathrm{~ns}$ ) for $\mathrm{pMLF} 1 / 14-3-3 \varepsilon$ and $\mathrm{npMLF} 1 / 14-3-3 \varepsilon$, respectively. A snapshot was stored every $1 \times 10^{5}$ steps (200 ps), yielding 153,600 and 71,680 snapshots for pMLF1/14-3-3 $\varepsilon$ and npMLF1/14-3-3e, respectively.

Here, we provide actual values of parameters which control the simulations. The atom groups adopted for the current study to define the three RCs are listed in Table S1. Also see Fig. 1 of main text, which graphically explains the atom groups and RCs. Table S2 lists parameters $n_{v s}(h)$, $\left[\zeta_{1}^{(h)}\right]_{\min },\left[\zeta_{n_{v s}(h)}^{(h)}\right]_{\max }$, and $\Delta \lambda^{(h)}$, and Table S3 does actual setting of zones: $\left\{\left[\lambda_{k}^{(h)}\right]_{\text {min }},\left[\lambda_{k}^{(h)}\right]_{\text {max }} ; k=1, \ldots, n_{v s}(h)\right\}$. An interzone transition was attempted once every 20 ps: The IVT transition interval was set to $1 \times 10^{4}$ steps. See Ref. 3 for meaning of parameters.

Table S1. Atom groups to define three RCs.

\section{Atom Group ${ }^{\text {a) }}$}

RC $\quad G_{h}^{A} \quad G_{h}^{B}$

$\begin{array}{lll}\lambda^{(\alpha)} & \text { Arg 130-Glu 134 }{ }^{\mathrm{b})} & \text { Arg 31 } \\ \lambda^{(\beta)} & \text { Leu 173-Val 179 } & \text { Sep 34/S } \\ \lambda^{(\gamma)} & \text { Ala 48-Val 52 } & \text { Phe 37 }\end{array}$

a) Two atom groups are expressed as $G_{h}^{A}$ or $G_{h}^{B}(h=\alpha, \beta, \gamma)$ to define $\lambda^{(h)}: \lambda^{(h)}$ is defined by the distance between the centers of mass of $G_{h}^{A}$ and $G_{h}^{B}$. Superscripts $A$ and $B$ are assigned to 14-3-3 $\varepsilon$ and pMLF1 (or npMLF1), respectively.

b) " $X n_{1}-Y n_{2}$ " represents all mainchain heavy and $\mathrm{C} \beta$ atoms from amino-acid $X$ at the $n_{1}$-th residue to amino-acid $Y$ at the $n_{2}$-th residue.

c) Sep 34 and Ser 34 are the phosphorylated serine of pMLF1 and the serine of npMLF1 at the 34-th residue, respectively.

Table S2. Parameters ${ }^{\text {a) }}$ for RC-space division 


$$
h \quad n_{v s}(h)\left[\zeta_{1}^{(h)}\right]_{\min } \quad\left[\zeta_{n_{v s}(h)}^{(h)}\right]_{\max } \quad \Delta \lambda^{(h)}
$$

$\begin{array}{lllll}\alpha & 16 & 6 \AA & 40.0 \AA & 4 \AA \\ \beta & 22 & 6 \AA & 40.5 \AA & 3 \AA \\ \gamma & 16 & 6 \AA & 40.0 \AA & 4 \AA\end{array}$

TableS3. Setting of zones.

Zone No. ${ }^{\text {a) }}$

$k \quad\left[\lambda_{k}^{(\alpha)}\right]_{\min }\left[\lambda_{k}^{(\alpha)}\right]_{\max }$

1

2

3

4

5

6

7

8

9

10

11

12

13

6.0

8.0

10.0

12.0

14.0

16.0

18.0

10.0

12.0

14.0

20.0

22.0

32.0

$30.0 \quad 34.0$
6.0

9.0

7.5

10.5

9.0

12.0

10.5

13.5

12.0

15.0

13.5

16.5

15.0

18.0

16.5

18.0

19.5

21.0

24.0

22.5

25.5

24.0

27.0

$\left[\lambda_{k}^{(\gamma)}\right]_{\min }\left[\lambda_{k}^{(\gamma)}\right]_{\max }{ }^{\mathrm{c})}$

24.0 


$\begin{array}{lllll}14 & 32.0 & 36.0 & 25.5 & 28.5 \\ 15 & 34.0 & 38.0 & 27.0 & 30.0 \\ 16 & 36.0 & 40.0 & 28.5 & 31.5 \\ 17 & & 30.0 & 33.0 \\ 18 & & 31.5 & 34.5 \\ 19 & & 33.0 & 36.0 \\ 20 & & 34.5 & 37.5 \\ 21 & & 36.0 & 39.0 \\ 22 & & & 37.5 & 40.5\end{array}$

a) Number of zones $n_{v s}$ is 16 for $\lambda^{(\alpha)}$ and $\lambda^{(\beta)}$, and 22 for $\lambda^{(\gamma)}$.

b) Unit of zones is $\AA$.

c) Parameters for $\lambda^{(\gamma)}$ are not shown because they are exactly the same as those for $\lambda^{(\alpha)}$. 


\section{Figure S6}

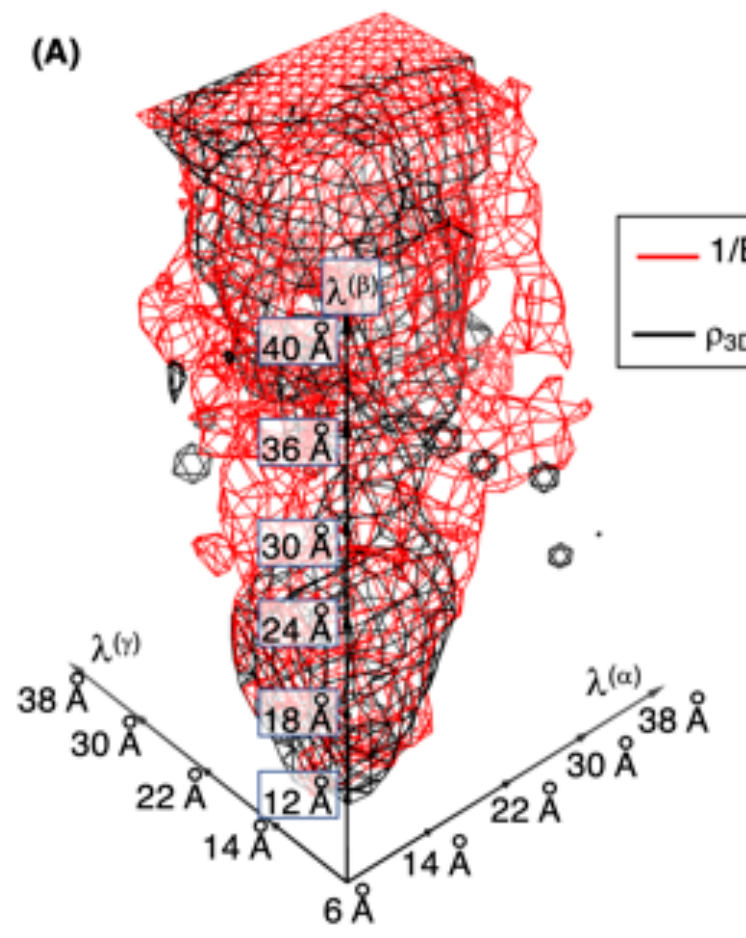

(B)
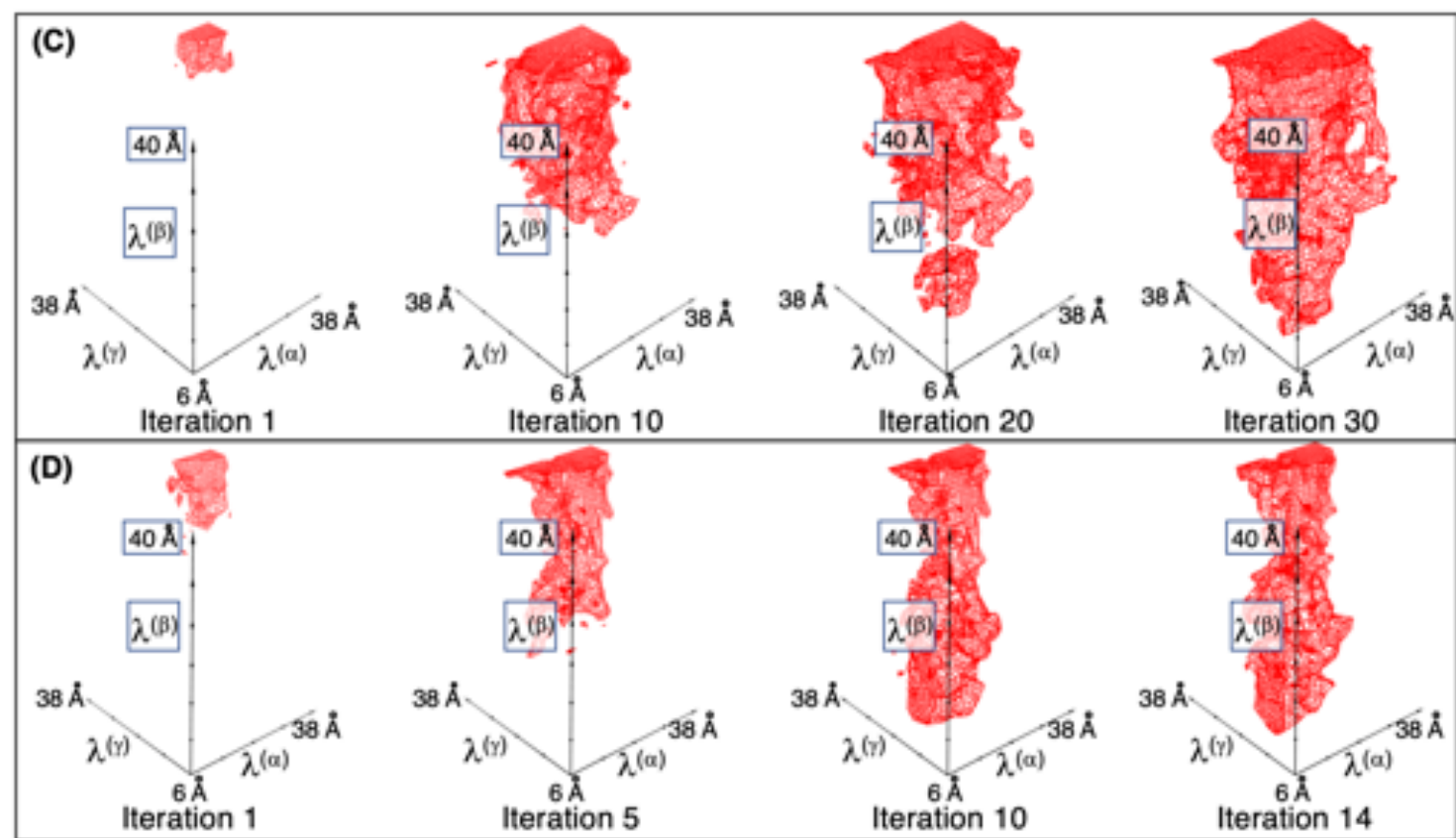

Figure S6.

Accuracy of obtained distribution $\rho_{3 D-R C}(\lambda)$ assessed by an objective function $E_{\text {local }}(\lambda)^{-1}$ introduced in our previous work. ${ }^{3}$ Benefit of this function is: The accuracy can be accessed locally in the RC space. The larger the $E_{\text {local }}(\lambda)^{-1}$ at a position $\lambda$, the more accurate the $\rho_{3 D-R C}(\lambda)$ at the position. This figure is presented at contour level of $E_{\text {local }}(\lambda)^{-1}=$

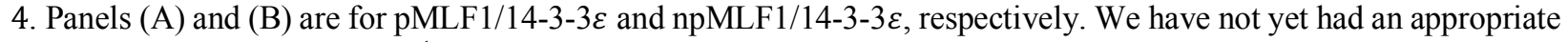
threshold to assess $E_{\text {local }}(\lambda)^{-1}$ because we have little experience with GA-guided VcMD. However, this figure indicated that regions, to which relatively large $\rho_{3 D-R C}(\lambda)$ are assigned $\left(\rho_{3 D-R C} \geq 0.1\right)$, are covered by contours of $E_{\text {local }}(\lambda)^{-1} \geq 4$. This is an expected result because the thermodynamically important RC regions should have more 
accuracy than the less important regions $\left(\rho_{3 D-R C}<0.1\right)$. Panels $(\mathrm{C})$ and (D) illustrate dependency of regions with $E_{\text {local }}(\lambda)^{-1} \geq 4$ on iteration No. for pMLF1/14-3-3 $\varepsilon$ and npMLF1/14-3-3 $\varepsilon$, respectively. Shown contours are $E_{\text {local }}(\lambda)^{-1} \geq 4$, which increases with proceeding iterations, and the contours covered the high-density regions $\left(\rho_{3 D-R C} \geq 0.1\right)$ in the 14-th and 30-th iterations for pMLF1/14-3-3 $\varepsilon$ and nPMLF1/14-3-3e, respectively.

\section{Figure S7}
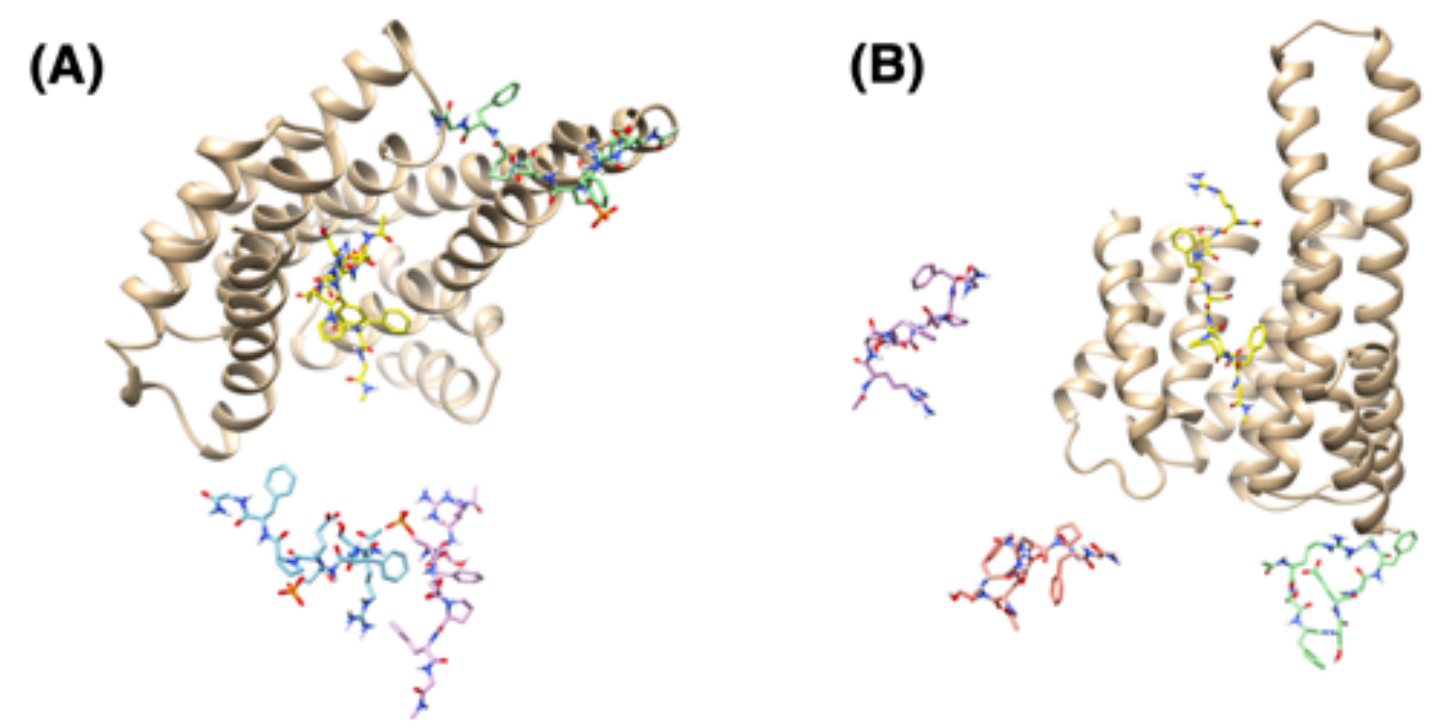

Figure S7. Panel (A) displays conformations taken randomly from cluster $C_{2}$ of Fig. $2 \mathrm{~A}$ for the pMLF1/14-3-3 $\varepsilon$ system, and panel (B) does those taken randomly from cluster $C_{2}^{\prime}$ of Fig. 2B for the npMLF1/14-3-3 $\varepsilon$ system. Yellowcolored MLF1 conformations in the panels are those from crystal structure.

\section{Figure S8}



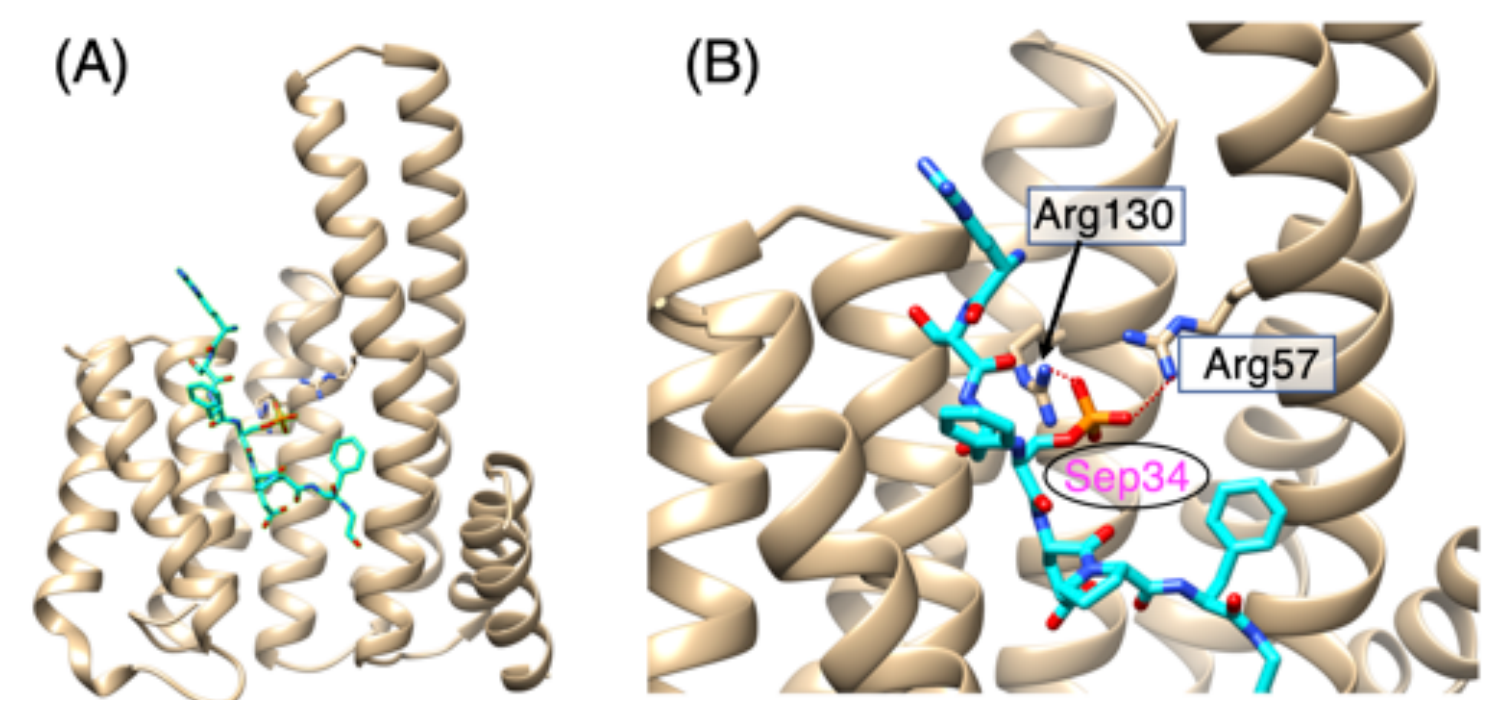

Figure S8. (A) pMLF1/14-3-3e complex to show the position of Sep 34 in the entire complex structure. pMLF1 is shown by cyan-colored model, and 14-3-3e by light yellow-colored model. (B) Close up of Sep 34 in pMLF1 and two residues Arg 57 and Arg 130 of 14-3-3e, which form salt-bridges (brown-colored broken lines).

\section{Section 5. Calculation of radial distribution function}

Here we present the method to calculate a radial distribution function. First, calculate a distance distribution function (or pair distance distribution function) $P\left(r^{\mu}\right)(\mu=$ Phe33, Sep 34/Ser 34, Glu35, or Phe37) from the equilibrated ensemble. A probability to detect the distance in a window $\left[r^{\mu}, r^{\mu}+d r^{\mu}\right]$ is given by $P\left(r^{\mu}\right) d r^{\mu}$. See main text for the definition of distances $r^{\mu}$. Then, a radial distribution function (or radial density function) was defined as: $p\left(r^{\mu}\right)=P\left(r^{\mu}\right) / 4 \pi\left(r^{\mu}\right)^{2}$ with normalization of $\int p\left(r^{\mu}\right) d r^{\mu}=1$.

\section{Section 6. Calculation of spatial density of pMLF1/npMLF1 around 14-3-3e}

First, we divide the 3D real space into cubes, whose size $\Delta V$ is $2 \AA \times 2 \AA \times 2 \AA$. The cube position is defined by its center $\boldsymbol{r}=[x, y, z]$. Then, we sum up the statistical weights of snapshots whose $\mathrm{O} \gamma$ atom of Sep 34 of pMLF1 (or Ser 34 of npMLF1) is detected in the cube. The sum of the weights is interpreted as a density at $\boldsymbol{r}$, and expressed as $\rho_{\operatorname{Sep} 34}^{O \gamma}(\boldsymbol{r})\left(\right.$ or $\left.\rho_{\operatorname{Ser} 34}^{O \gamma}(\boldsymbol{r})\right)$. Last, we normalize the function as $\sum_{i} \rho_{\mu}^{O \gamma}\left(\boldsymbol{r}_{i}\right)=1$, where the summation is taken over all cubes $(\forall i)$. This normalization makes the density map comparable between the pMLF1/14-3-3 $\varepsilon$ and npMLF1/143-3 $\varepsilon$ systems.

Other spatial density functions were calculated with the same manner. For instance, a density, $\rho_{R}^{a}(\boldsymbol{r})$, for the position of an atom $a$ of residue $R$ is calculated by summing up the statistical weights of snapshots whose atom $a$ is detected in the cube $\boldsymbol{r}$. We use this spatial distribution function to analyze the distribution of Sep/Ser 34 and Glu 35 in this study. To analyze 
the density of Phe 33 and Phe 37, we calculated the position of the sidechain ring center of Phe 33 or Phe 37, and calculated the density functions for the ring centers: $\rho_{P h e 33}^{\text {ring }}(\boldsymbol{r})$ and $\rho_{P h e 37}^{\text {ring }}(\boldsymbol{r})$.

\section{Figure S9}

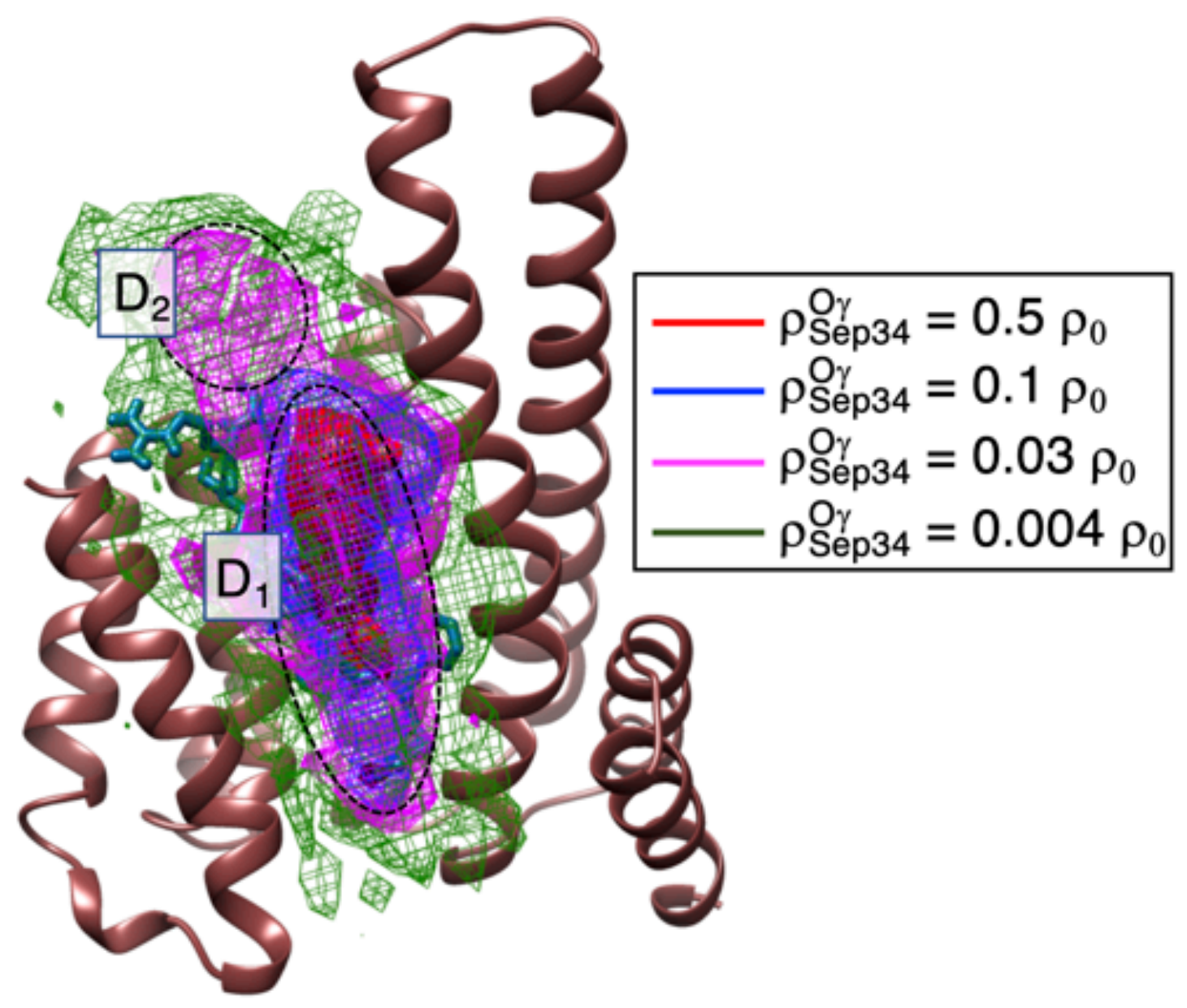

Figure S9. Distribution $\rho_{\text {Sep34 }}^{o \gamma}(\boldsymbol{r})$ of the pMLF1/14-3-3E system using snapshots in cluster $C_{1}$, which was introduced in Fig. 2A of main text. Selection of snapshots from $C_{1}$ was done as follows: Full variable ranges of RCs for this simulation are [6 $6,40 \AA]$, [6 $2,40.5 \AA]$, and [6 $\mathrm{6}, 40 \AA]$ for $\lambda_{1}, \lambda_{2}$, and $\lambda_{3}$, respectively (see Table $\mathrm{S} 3$ of SI). We verified that restricted ranges $6 \AA \leq \lambda_{1} \leq 20 \AA, 6 \AA \leq \lambda_{2} \leq 20.25 \AA$, and $6 \AA \leq \lambda_{1} \leq 20 \AA$ do not involve snapshots in cluster $C_{2}$. Thus, this figure was generated using the snapshots in the restricted ranges. This means that this figure was contributed by snapshots in intermediate regions other than $C_{1}$ or $C_{2}$. However, such conformations have a very small thermodynamics weights and do not contribute substantially to the shown distribution.

Figure S10 
bioRxiv preprint doi: https://doi.org/10.1101/2020.05.24.113209; this version posted August 20, 2020. The copyright holder for this preprint (which was not certified by peer review) is the author/funder, who has granted bioRxiv a license to display the preprint in perpetuity. It is made available under aCC-BY-NC-ND 4.0 International license.

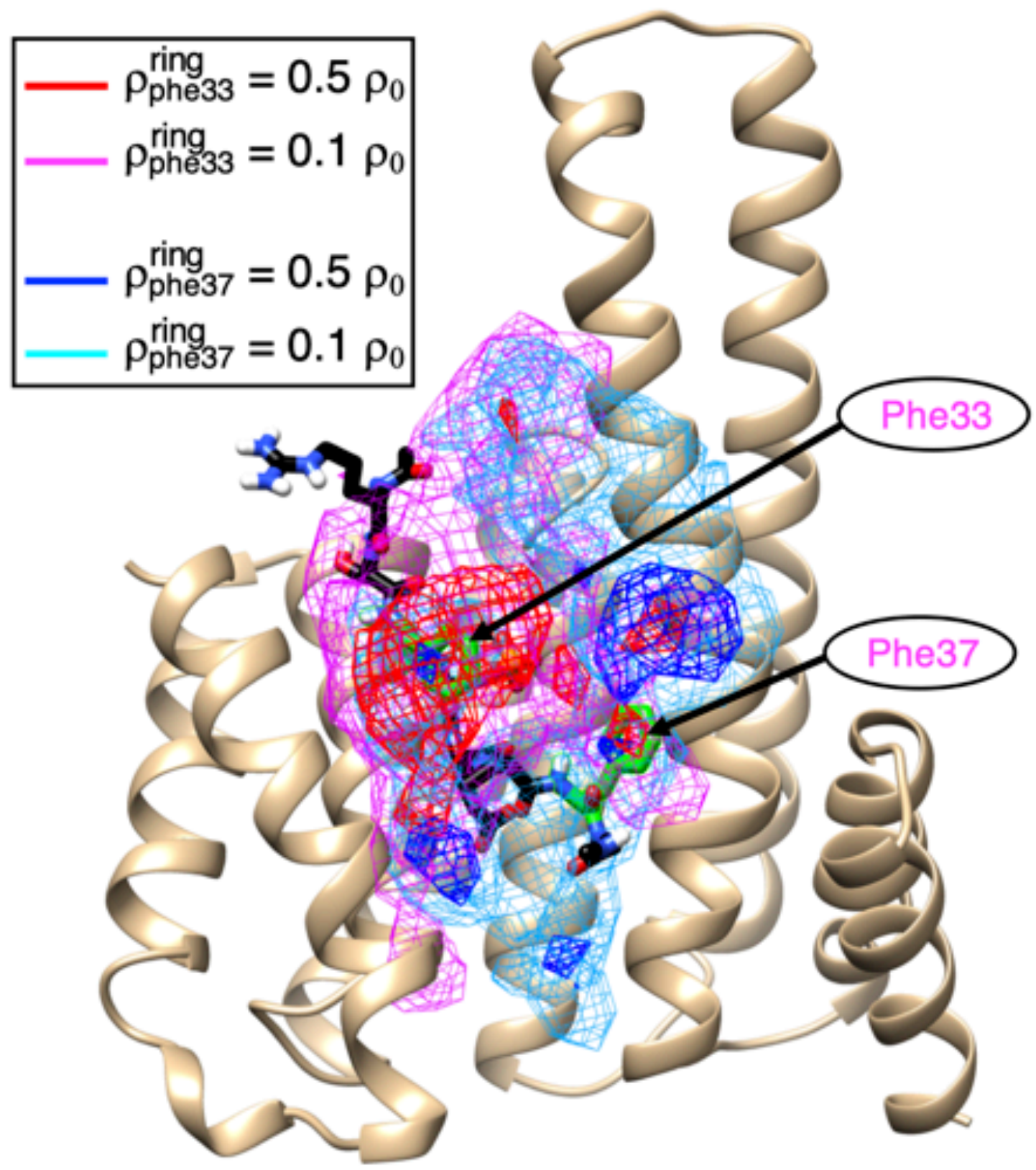

Figure S10. Distribution of $\rho_{\text {phe33 }}^{\text {ring }}$ and $\rho_{\text {phe37 }}^{\text {ring }}$ of the pMLF1/14-3-3e system using snapshots only from cluster $C_{1}$. See caption of Fig. $\mathrm{S} 7$ for selection of snapshots from $C_{1}$.

Figure S11 


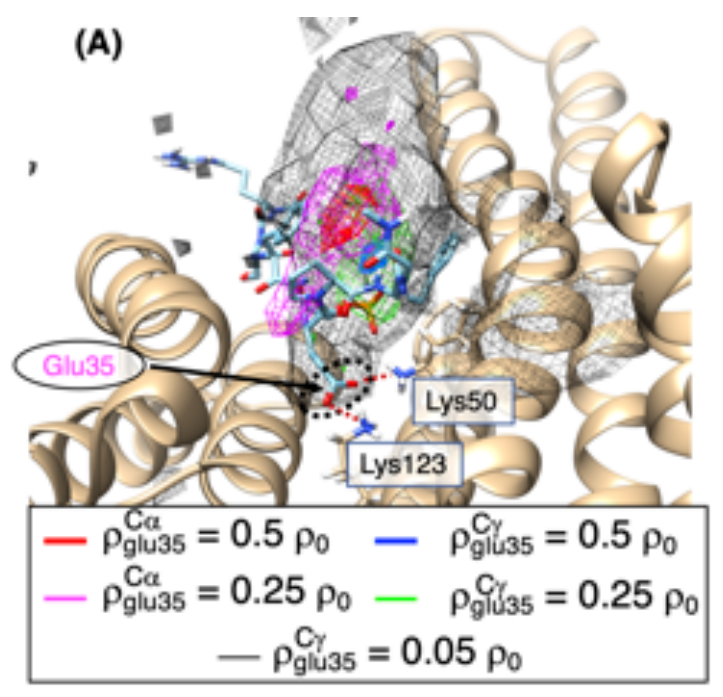

(B)

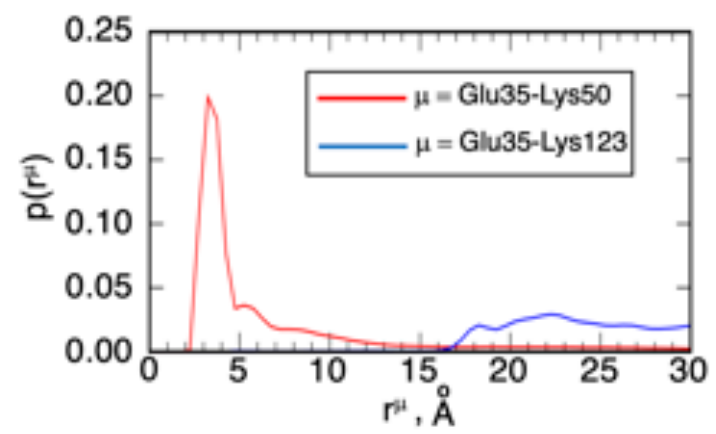

Figure S11. (A) Density contour maps of $\rho_{G l u 35}^{C \alpha}(\boldsymbol{r})$ and $\rho_{G l u 35}^{C \gamma}(\boldsymbol{r})$ for pMLF1/14-3-3e. Shown molecular structure is the crystal one focusing on Glu 35 of pMLF1. Contour levels are shown in inset $\left(\rho_{0}=0.01\right)$. Black-colored contours $\left(\rho_{\text {Glu }}^{\text {Cr }}(\boldsymbol{r})=0.05 \rho_{0}\right)$ cover the sidechain-tip position of Glu 35 of the structure, where Glu 35 forms salt bridges (brown-colored broken line) to both Lys 50 and Lys 123 of 14-3-3e. Black broken-line circle is mentioned in main text. (B) Radial distribution function, $p\left(r^{G l u 35-L y s 50}\right)$, for distance $r^{G l u 35-L y s 50}$ from the $\mathrm{C} \gamma$ atom of Glu 35 of pMLF1 to the $\mathrm{N} \zeta$ atom of Lys 50 of 14-3-3 $\varepsilon$, and radial distribution function $p\left(r^{G l u 35-L y s 123}\right)$ for distance

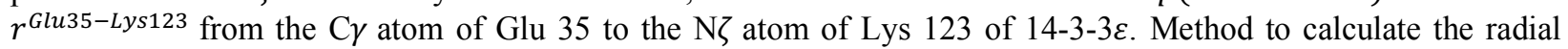
distribution function is given in section 5 of SI.

\section{Section 7. Salt-bridge-formation probabilities}

The salt-bridge-formation probabilities are defined by $Q_{G l u 35-L y s 50}=\sum_{i}^{r^{G l u 35-L y s 50} \leq 5.5 \AA} w_{i}$ and $Q_{\text {Glu35-Lys123 }}=\sum_{i}^{r^{G l u 35-L y s 123} \leq 5.5 \AA} W_{i}$, respectively. $r^{G l u 35-L y s 50}$ is the distance from the $\mathrm{C} \gamma$

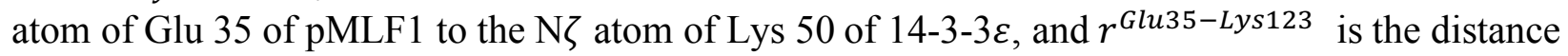
from the $\mathrm{C} \gamma$ atom of Glu 35 to the $\mathrm{N} \zeta$ atom of Lys 123 of 14-3-3e. The $w_{i}$ is the statistical weight assigned to snapshot $i$. The summation for $Q_{G l u 35-L y s 50}$ is taken over snapshots with $r^{\text {Glu35-Lys50 }} \leq 5.5 \AA$ and that for $Q_{\text {Glu35-Lys } 123}$ is done over those with $r^{\text {Glu35-Lys } 123} \leq 5.5 \AA$. One may consider that the value of $5.5 \AA$ is larger than a limit distance for a salt bridge. However, the $\mathrm{C} \gamma$ atom, not $\mathrm{O} \varepsilon$ atoms, was used to calculate $r^{\text {Glu35-Lys50 }}$ and $r^{\text {Glu35-Lys123, and Glu } 35 \text { has }}$ large positional fluctuations as shown above: Remember that a snapshot was saved once every 200 ps. Thus, we increased the limit distance.

\section{Section 8. Average of the orientation at each cube}

In the main text, the orientation vector $\boldsymbol{e}_{i}\left(\left|\boldsymbol{e}_{i}\right|=1\right)$ was defined for snapshot $i$. Here, we present a method for averaging the orientation vectors in each cube. As done for calculating the spatial density function (see section 6 of SI), we divided the $3 \mathrm{D}$ real space into cubes of $2.0 \AA \times 2.0 \AA \times 2.0 \AA$, whose positions are assigned to the cube centers $\boldsymbol{r}$. A snapshot was 
assigned to a cube when the $\mathrm{O} \gamma$ atom of Sep 34 of the snapshot is involved in the cube. Then, average of orientation vectors is taken for each cube:

$$
\overline{\boldsymbol{v}}(\boldsymbol{r})=\frac{\sum_{i} \delta_{c u b e}(\boldsymbol{r} ; i) w_{i} \boldsymbol{e}_{i}}{\sum_{i} \delta_{c u b e}(\boldsymbol{r} ; i) w_{i}}
$$

where $\delta_{\text {cube }}(\boldsymbol{r} ; i)$ is a delta function defined as:

$$
\delta_{c u b e}(\boldsymbol{r} ; i)=\left\{\begin{array}{lr}
1 & \text { (if } 0 \gamma \text { atom of snapshot } i \text { is in cube at } \boldsymbol{r}) \\
0 & \text { (else) }
\end{array} .\right.
$$

The quantity $\sum_{i} \delta_{\text {cube }}(\boldsymbol{r} ; i) w_{i}$ in Eq. S1 is equivalent to $\rho_{\text {Sep34 }}^{O \gamma}(\boldsymbol{r})$. Note that $\overline{\boldsymbol{v}}(\boldsymbol{r})$ is no longer a unit vector. The norm of vector $|\overline{\boldsymbol{v}}(\boldsymbol{r})|$ indicates a degree of orientational ordering of pMLF1 detected in cube $\boldsymbol{r}:|\bar{v}(\boldsymbol{r})|$ takes the maximum $(=1.0)$ when all $\boldsymbol{e}_{i}$ have exactly the same orientations in the cube. If snapshots have uncorrelated orientations, then $|\bar{v}(\boldsymbol{r})|$ becomes small.

\section{Section 9. Ligand-conformational variety of pMLF1 at each cube}

Here we present the procedure to calculate the ligand-conformational variety, $\Delta r_{P h e-P h e}(\boldsymbol{r})$, of pMLF1 at each cube $r$. First, we introduce a distance $r_{P h e-P h e}$, which is the $\mathrm{C} \alpha$ atomic distance between Phe 33 and Phe 37. The value of $r_{P h e-P h e}$ for the crystal structure was $11.726 \AA$. Then we represent the ligand-conformational variety $\Delta r_{P h e-P h e}$ by the standard deviation of $r_{P h e-P h e}$ in each cube $\boldsymbol{r}$ :

$$
\Delta r_{P h e-P h e}(\boldsymbol{r})=\left[\frac{\sum_{i} \delta_{c u b e}(\boldsymbol{r} ; i) r_{P h e-P h e}{ }^{2} w_{i}}{\sum_{i} \delta_{c u b e}(\boldsymbol{r} ; i) w_{i}}-\left(\frac{\sum_{i} \delta_{c u b e}(\boldsymbol{r} ; i) r_{P h e-P h e} w_{i}}{\sum_{i} \delta_{c u b e}(\boldsymbol{r} ; i) w_{i}}\right)^{2}\right]^{1 / 2}
$$

The larger the $\Delta r_{P h e-P h e}(\boldsymbol{r})$ in a cube $\boldsymbol{r}$, the larger the ligand-conformational variety in the cube. We note that $\Delta r_{P h e-P h e}(\boldsymbol{r})$ is not directly linked to a dynamic chain flexibility of pMLF1 because the conformational ensemble from the GA-guided $\mathrm{mD}-\mathrm{VcMD}$ is an equilibrated one at $300 \mathrm{~K}$.

\section{Figure S12}




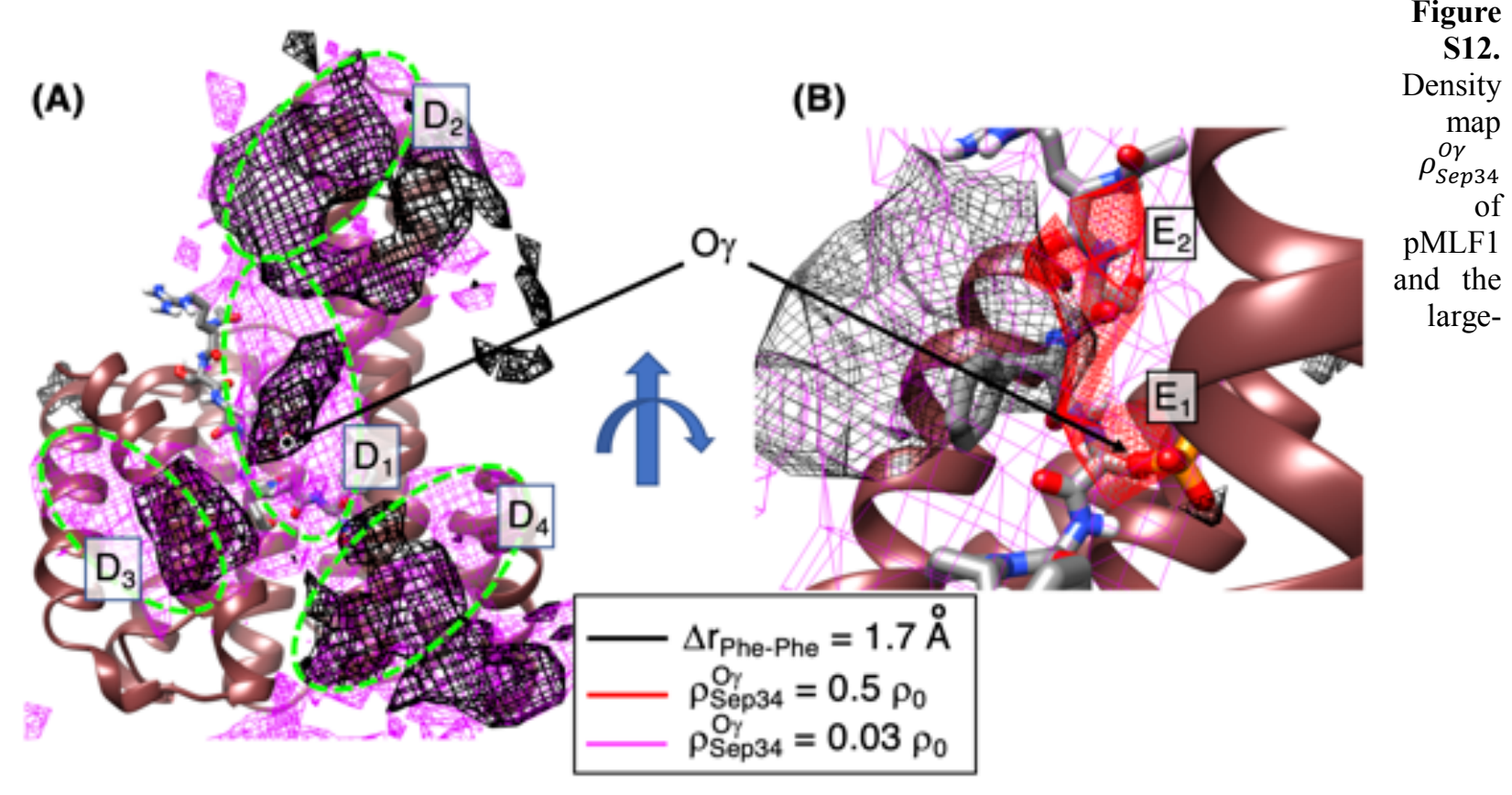

$\Delta r_{\text {Phe-Phe }}$ contours. Panel A, which is the same as Fig. 9B of main text except for the green-colored contours of Fig. 9B, shows clusters $D_{1}, \ldots, D_{4}$. Panel B is a side view of panel A with focusing cluster $D_{1}$. High-density spots $E_{1}$ and $E_{2}$ were defined in Fig. 6 of main text. Both panels present the position of $O_{\gamma}$ atom of Sep 34 in pMLF1.

\section{Section 10. Thermal average of distances in each cube}

Here, we define a procedure to calculate thermal average of distances $r^{\mu}(\mu=$ Phe33, Sep 34, Glu35, Phe37) by the following equation:

$$
\bar{r}^{\mu}(\boldsymbol{r})=\frac{\sum_{i} \delta_{\text {cube }}(\boldsymbol{r} ; i) w_{i} r^{\mu}(i)}{\sum_{i} \delta_{\text {cube }}(\boldsymbol{r} ; i) w_{i}}
$$

Where $r^{\mu}(i)$ is $r^{\mu}$ of snapshot $i$. The delta function $\delta_{\text {cube }}$ (Eq. S2 in SI) works so that only snapshots, whose $\mathrm{O} \gamma$ atom of Sep 34 are detected in the cube at $\boldsymbol{r}$, contribute to $\bar{r}^{\mu}(\boldsymbol{r})$. The $\bar{r}^{\mu}(\boldsymbol{r})$ quantifies space dependency of the contacts of the four residues (Phe 33, Sep 34, Glu 35, and Phe 37) of pMLF1 to their contact partners of $14-3-3 \varepsilon$ as a function of $\boldsymbol{r}$. The smaller the distance $r^{\mu}$ in a cube $\boldsymbol{r}$, the contact tends to be formed in the cube.

\section{References}

[1] Kawabata, T.; Nishikawa, K. Protein structure comparison using the Markov transition model of evolution. Proteins 2000, 41, 108-122. 
[2] Molzan, M.; Weyand, M.; Rose, R.; Ottmann, C. Structural Insights of the MLF1/14-3-3 Interaction. FEBS J. 2012, 279, 563-571.

[3] Higo, J.; Kusaka, A.; Kasahara, K.; Kamiya, N.; Fukuda, I.; Mori, K.; Hata, Y.; Fukunishi, F. arXiv: 2006.06950, 2020. 